\title{
The Abel-Jacobi map for higher Chow groups
}

\author{
Matt Kerr, James D. Lewis and Stefan Müller-Stach \\ In memory of Fabio Bardelli
}

\begin{abstract}
We construct a map between Bloch's higher Chow groups and Deligne homology for smooth, complex quasiprojective varieties on the level of complexes. For complex projective varieties this results in a formula which generalizes at the same time the classical Griffiths Abel-Jacobi map and the Borel/Beilinson/Goncharov regulator type maps.
\end{abstract}

\section{Introduction}

Let $C H^{\bullet}(X, \bullet)$ be the higher Chow groups as introduced by Bloch [Blo86a], and let $H_{\mathcal{D}}^{\bullet}(X, \mathbb{Z}(\bullet))$ be Deligne cohomology. Bloch [Blo86b] constructed, for $X$ smooth, a cycle-class map

$$
c_{p, n}: C H^{p}(X, n) \rightarrow H_{\mathcal{D}}^{2 p-n}(X, \mathbb{Z}(p)) .
$$

A somewhat different, but equivalent, approach using extension classes, is presented in [DS91] and [Sch93]. The purpose of this paper is to give an explicit description of this map in terms of currents. More specifically, we are interested in the case where $X$ is a smooth projective variety defined over $\mathbb{C}$, and the higher cycle group in question is that of the nullhomologous cycles $C H_{\text {hom }}^{p}(X, n)$. The results of this paper pertain to this restricted setting.

As suggested by the title of this paper, we are generalizing the classical Abel-Jacobi map involving a membrane integral (Griffiths' prescription) and the Borel/Beilinson/Goncharov regulator type maps involving multiple logarithms, to higher Chow groups. To state this more precisely, we recall that there is a short exact sequence:

$$
\begin{aligned}
0 \rightarrow \frac{H^{2 p-n-1}(X, \mathbb{C})}{F^{p} H^{2 p-n-1}(X, \mathbb{C})+H^{2 p-n-1}(X, \mathbb{Z}(p))} & \rightarrow H_{\mathcal{D}}^{2 p-n}(X, \mathbb{Z}(p)) \\
& \rightarrow H^{2 p-n}(X, \mathbb{Z}(p)) \cap F^{p} \rightarrow 0 .
\end{aligned}
$$

Put

$$
C H_{\text {hom }}^{p}(X, n)=\operatorname{ker}\left\{C H^{p}(X, n) \rightarrow H_{\mathcal{D}}^{2 p-n}(X, \mathbb{Z}(p)) \rightarrow H^{2 p-n}(X, \mathbb{Z}(p))\right\} .
$$

Definition 1.1. The induced map

$$
\Phi_{p, n}: C H_{\mathrm{hom}}^{p}(X, n) \rightarrow \frac{H^{2 p-n-1}(X, \mathbb{C})}{F^{p} H^{2 p-n-1}(X, \mathbb{C})+H^{2 p-n-1}(X, \mathbb{Z}(p))}
$$

is called the Abel-Jacobi map.

Received 4 November 2004, accepted in final form 22 June 2005.

2000 Mathematics Subject Classification 14C25, 14C30, 14C35, 19E15.

Keywords: Abel-Jacobi map, regulator, Deligne cohomology, higher Chow group.

The second author was partially supported by a grant from the Natural Sciences and Engineering Research Council of Canada. The third author was supported by a startup grant from McMaster University.

This journal is (c) Foundation Compositio Mathematica 2006. 
Let $m=\operatorname{dim} X$. By Poincaré and Serre duality, we will think of this map in the form

$$
\Phi_{p, n}: C H_{\mathrm{hom}}^{p}(X, n) \rightarrow \frac{\left\{F^{m-p+1} H^{2 m-2 p+n+1}(X, \mathbb{C})\right\}^{\vee}}{H_{2 m-2 p+n+1}(X, \mathbb{Z}(m-p))} .
$$

We will use the cubical complex description of $C H^{p}(X, n)$ throughout this paper (see $\S 2$ ). Cycles live in $X \times \square^{n}$, where $\square^{n}:=\left(\mathbb{P}_{\mathbb{C}}^{1} \backslash\{1\}\right)^{n}$ has coordinates $\left(z_{1}, \ldots, z_{n}\right)$, and there are projection maps $\pi_{X}: X \times \square^{n} \rightarrow X$ and $\pi_{\square}: X \times \square^{n} \rightarrow \square^{n}$. Consider an irreducible subvariety $\mathcal{Z} \subset X \times \square^{n}$, of codimension $p$, and a form $\omega \in F^{m-p+1} \Omega_{X^{\infty}}^{2 m-2 p+n+1}(X)$.

One considers the current associated to $\mathcal{Z}$, defined by its action on $\omega$ :

$$
\begin{aligned}
\frac{1}{(2 \pi \mathrm{i})^{n-p}}[ & \int_{\mathcal{Z} \backslash\left\{\mathcal{Z} \cap \pi_{\square}^{-1}\left([-\infty, 0] \times \square^{n-1}\right)\right\}} \pi_{\square}^{*}\left(\left(\log z_{1}\right) \operatorname{dlog} z_{2} \wedge \cdots \wedge \operatorname{dlog} z_{n}\right) \wedge \pi_{X}^{*} \omega \\
& +(-2 \pi \mathrm{i}) \int_{\substack{\left.\left\{\mathcal{Z} \cap \pi_{\square}^{-1}[-\infty, 0] \times \square^{n-1}\right\} \\
\square^{n-1}\left([-\infty, 0]^{2} \times \square^{n-2}\right)\right\}}} \pi_{\square}^{*}\left(\left(\log z_{2}\right) \operatorname{dlog} z_{3} \wedge \cdots \wedge \operatorname{dlog} z_{n}\right) \wedge \pi_{X}^{*} \omega+\cdots \\
& +(-2 \pi \mathrm{i})^{n-1} \int_{\left\{\mathcal{Z} \cap \pi_{\square}^{-1}\left([-\infty, 0]^{n-1} \times \square\right)\right\}} \pi_{\square}^{*}\left(\log z_{n}\right) \wedge \pi_{X}^{*} \omega \\
& \left.+(-2 \pi \mathrm{i})^{n} \int_{\zeta} \pi_{X}^{*} \omega\right] .
\end{aligned}
$$

Here the latter term is a membrane integral, and $\log z_{i}$ represents the branch of the logarithm with argument in $(-\pi, \pi)$ (same for every $z_{i}$ ).

TheOrem 1.2. The map $\Phi_{p, n}$ is induced by the above current.

Remark. Strictly speaking, in terms of homology the Tate twist should be $1 /(2 \pi i)^{m-p+n}$ in the above formula. However, we felt it was more natural to give a cohomological formulation of the map, where the extra $(2 \pi \mathrm{i})^{m}$ is lost in passage from Deligne homology to cohomology, as indicated in $\S 5.6$.

The plan of the paper is as follows. After reviewing the classical situation $(n=0)$, we arrive at the formula for the Abel-Jacobi $(A J)$ map, based on a cup-product calculation at the generic point. This was the point of view adopted by the second author. Around the same time the first author arrived at the formula based on a morphism of complexes. This is fully explained in [Ker03], and the relevant points are explained here. By comparing the extension class [Sch93] construction with the above formula, we arrive at the above theorem. We are grateful to S. Bloch for remarking that the $A J$ map can be described in terms of the dilogarithm associated to the 'Totaro' cycles that are discussed in [Blo91]. This led to the inclusion of an example in $\S 5.7$ below.

\section{Some definitions}

\subsection{Higher Chow groups}

A reference for this section is [Blo86a]. Let $W / \mathbb{C}$ be a quasiprojective variety. Denote by $Z^{k}(W)$ the free abelian group generated by subvarieties of codimension $k$ in $W$. Consider the $n$-simplex

$$
\Delta^{n}=\operatorname{Spec}\left\{\frac{\mathbb{C}\left[t_{0}, \ldots, t_{n}\right]}{\left(1-\sum_{j=0}^{n} t_{j}\right)}\right\} \simeq \mathbb{C}^{n} .
$$

We set

$$
\begin{array}{r}
Z^{k}(W, n):=\left\{\xi \in Z^{k}\left(W \times \Delta^{n}\right) \mid \text { every component of } \xi\right. \text { meets } \\
\text { all faces } \left.\left\{t_{i_{1}}=\cdots=t_{i_{\ell}}=0, \ell \geqslant 1\right\} \text { properly }\right\}
\end{array}
$$




\section{KerR, J. D. Lewis ANd S. MÜLler-Stach}

Note that $Z^{k}(X, 0)=Z^{k}(W)$. Now set $\partial_{j}: Z^{k}(W, n) \rightarrow Z^{k}(W, n-1)$ to be the restriction to the $j$ th face (given by $t_{j}=0$ ). The boundary map

$$
\delta=\sum_{j=0}^{n}(-1)^{j} \partial_{j}: Z^{k}(W, n) \rightarrow Z^{k}(W, n-1)
$$

satisfies $\delta^{2}=0$.

Definition 2.1. From [Blo86a], $C H^{k}(W, \bullet)=$ homology of $\left\{Z^{k}(W, \bullet), \delta\right\}$. We put $C H^{k}(W):=$ $C H^{k}(W, 0)$.

Cubical version. Let $\square^{n}:=\left(\mathbb{P}^{1} \backslash\{1\}\right)^{n}$ with coordinates $z_{i}$ and $2^{n}$ codimension-one faces obtained by setting $z_{i}=0, \infty$, and boundary maps $\partial=\sum(-1)^{i-1}\left(\partial_{i}^{0}-\partial_{i}^{\infty}\right)$, where $\partial_{i}^{o}, \partial_{i}^{\infty}$, denote the restriction maps to the faces $z_{i}=0, z_{i}=\infty$, respectively. The rest of the definition is completely analogous except that one has to divide out degenerate cycles. The precise description is given in $\S 5.2$. It is known that both complexes are quasiisomorphic.

\subsection{Deligne cohomology}

Working in the analytic topology, we introduce the Deligne complex (for any subring $\mathbb{A} \subseteq \mathbb{R}$ )

$$
\mathbb{A}_{\mathcal{D}}(k): \mathbb{A}(k) \rightarrow \underbrace{\mathcal{O}_{X} \rightarrow \Omega_{X}^{1} \rightarrow \cdots \rightarrow \Omega_{X}^{k-1}}_{\text {call this } \Omega_{X}^{\bullet<k}} .
$$

Definition 2.2. Deligne cohomology is given by the hypercohomology

$$
H_{\mathcal{D}}^{i}(X, \mathbb{A}(k))=\mathbb{H}^{i}\left(\mathbb{A}_{\mathcal{D}}(k)\right) .
$$

From the short exact sequence

$$
0 \rightarrow \Omega_{X}^{\bullet<k}[-1] \rightarrow \mathbb{A}_{\mathcal{D}}(k) \rightarrow \mathbb{A}(k) \rightarrow 0,
$$

one has the short exact sequence

$$
\begin{aligned}
0 \rightarrow \frac{H^{i-1}(X, \mathbb{C})}{H^{i-1}(X, \mathbb{A}(k))+F^{k} H^{i-1}(X, \mathbb{C})} & \rightarrow H_{\mathcal{D}}^{i}(X, \mathbb{A}(k)) \\
& \rightarrow H^{i}(X, \mathbb{A}(k)) \cap F^{k} H^{i}(X, \mathbb{C}) \rightarrow 0 .
\end{aligned}
$$

In particular, the case $(\mathbb{A}, i, k)=(\mathbb{Z}, 2 p-n, p)$ gives the exact sequence in the introduction.

\subsection{Deligne homology}

In this part, we follow [Jan88] rather closely. Let $\mu: A^{\bullet} \rightarrow B^{\bullet}$ be a morphism of complexes. Then the cone complex is given by

$$
\operatorname{Cone}\left(A^{\bullet} \stackrel{\mu}{\rightarrow} B^{\bullet}\right):=A^{\bullet}[1] \oplus B^{\bullet},
$$

where the differential $\delta: A^{\bullet+1} \oplus B^{\bullet} \rightarrow A^{\bullet+2} \oplus B^{\bullet}$ is given by $\delta(a, b)=(-d a, \mu(a)+d b)$.

We introduce some notation: $\Omega_{X^{\infty}}^{p, q}$ is the sheaf of $C^{\infty}(p, q)$-forms on $X ;{ }^{\prime} \Omega_{X^{\infty}}^{p, q}$ is the sheaf of distributions over $\Omega_{X^{\infty}}^{-p,-q}$. Thus for an open set $U \subset X$, an element of ' $\Omega_{X^{\infty}}^{p, q}(U)$ is a continuous linear functional on the compactly supported forms $\Gamma_{c}\left(U, \Omega_{X^{\infty}}^{-p,-q}\right)$.

Key example I. Any $C^{\infty}(p, q)$-form $\eta$ gives a section of ${ }^{\prime} \Omega_{X}^{p-m, q-m}$ by the formula

$$
\omega \mapsto l(\eta)(\omega)=\frac{1}{(2 \pi \mathrm{i})^{m}} \int_{X} \eta \wedge \omega .
$$




\section{The ABel-JACOBI MAP FOR Higher Chow Groups}

Key example II. Any piecewise smooth oriented topological $r$-chain $\xi$ on $X$ gives a current $\delta_{\xi}$ in $\bigoplus_{p+q=-r}{ }^{\prime} \Omega_{X^{\infty}}^{p, q}(X)$ by the formula

$$
\omega \mapsto \epsilon(\xi)(\omega)=\int_{\xi} \omega
$$

Sheaves ${ }^{\prime} \Omega_{X^{\infty}}^{\bullet \bullet \bullet}$ and $\Omega_{X^{\infty}}^{\bullet \bullet \bullet}$ naturally form double complexes, where if $D$ is a current acting on an $r$-form, and given an $(r-1)$-form $\omega$, then $d D(\omega)=(-1)^{r-1} D(d \omega), d=\partial+\bar{\partial}$. [Warning: The definition of $d D$ differs from that in [Jan88] by a minus sign.] One has Hodge filtrations

$$
F^{i} \Omega_{X^{\infty}}^{\bullet}=\bigoplus_{p+q=\bullet, p \geqslant i} \Omega_{X^{\infty}}^{p, q}, \quad F^{i \prime} \Omega_{X^{\infty}}^{\bullet}=\bigoplus_{p+q=\bullet, p \geqslant i}{ }^{\prime} \Omega_{X^{\infty}}^{p, q} .
$$

Let $\left(C_{\bullet}(X, \mathbb{Z}(k)), d\right)$ be the complex of singular $C^{\infty}$-chains with coefficients in $\mathbb{Z}(k)$, and put ${ }^{\prime} C^{i}=C_{-i}\left[\right.$ with differential $\left.(-1)^{i+1} d: C_{-i} \rightarrow C_{-i-1}\right]$. One has a morphism of complexes

$$
\epsilon:{ }^{\prime} C^{\bullet}(X, \mathbb{Z}(k)) \rightarrow{ }^{\prime} \Omega^{\bullet}(X) .
$$

Put

$$
M_{\mathcal{D}}^{\bullet}=\operatorname{Cone}\left\{{ }^{\prime} C^{\bullet}(X, \mathbb{Z}(p-m)) \oplus F^{p-m}{ }^{\prime} \Omega_{X^{\infty}}^{\bullet}(X) \stackrel{\epsilon-l}{\longrightarrow}{ }^{\prime} \Omega_{X^{\infty}}^{\bullet}(X)\right\}[-1] .
$$

The homology of this complex, at $\bullet=2 p-n-2 m$, viz., ' $H_{\mathcal{D}}^{2 p-n-2 m}(X, \mathbb{Z}(p-m))$, is precisely the Deligne homology

$$
H_{2 m-2 p+n}^{\mathcal{D}}(X, \mathbb{Z}(m-p)):={ }^{\prime} H_{\mathcal{D}}^{2 p-n-2 m}(X, \mathbb{Z}(p-m)) \simeq H_{\mathcal{D}}^{2 p-n}(X, \mathbb{Z}(p))
$$

(Poincaré duality).

Remark 2.3. A class in $H_{2 m-2 p+n}^{\mathcal{D}}(X, \mathbb{Z}(m-p))$ is represented by a triple

$$
(a, b, c) \in{ }^{\prime} C^{2 p-n-2 m}(X, \mathbb{Z}(p-m)) \oplus F^{p-m}{ }^{2} \Omega_{X^{\infty}}^{2 p-n-2 m}(X) \oplus^{\prime} \Omega_{X^{\infty}}^{2 p-n-2 m-1}(X),
$$

where $d a=0, d b=0$, and $a-b+d c=0$. Via Poincaré duality, this corresponds to $[a] \in$ $H^{2 p-n}(X, \mathbb{Z}(p)),[b] \in F^{p} H_{\mathrm{DR}}^{2 p-n}(X, \mathbb{C})$, with $[a]=[b]$ in $H^{2 p-n}(X, \mathbb{C})$. Now suppose that $[a]=$ $[b]=0$. Then $a=d a_{0}$, and from Hodge theory, $b=d b_{0}$, where $b_{0} \in F^{p-m} \Omega_{X^{\infty}}^{2 p-n-2 m-1}(X)$. Thus $d\left(a_{0}-b_{0}+c\right)=0$, and $\left[a_{0}-b_{0}+c\right]$ represents the corresponding class in

$$
\frac{H^{2 p-n-1}(X, \mathbb{C})}{F^{p} H^{2 p-n-1}(X, \mathbb{C})+H^{2 p-n-1}(X, \mathbb{Z}(p))} \simeq \frac{F^{m-p+1} H^{2 m+n-2 p+1}(X, \mathbb{C})^{\vee}}{H_{2 m+n-2 p+1}(X, \mathbb{Z}(m-p))} .
$$

By Hodge-type considerations, the action of the current $b_{0}$ on $F^{m-p+1} H^{2 m+n-2 p+1}(X, \mathbb{C})$ is zero. Thus the action of the closed current $a_{0}-b_{0}+c$ on $F^{m-p+1} H^{2 m+n-2 p+1}(X, \mathbb{C})$ is the same as the action of $a_{0}+c$.

We also need a slightly expanded version of Deligne homology for the smooth quasiprojective case. Let $Z$ be a smooth quasiprojective with good compactification $\bar{Z}$ (with normal crossing divisor $E)$. Then $C_{\bullet}(\bar{Z}, \mathbb{A}(k))$ is the complex of singular $C^{\infty}$-chains in $\bar{Z}$ with coefficients in $\mathbb{A}(k)$, and ${ }^{\prime} C^{i}=C_{-i}$. Let

$$
{ }^{\prime} C^{\bullet}(\bar{Z}, E, \mathbb{A}(k))={ }^{\prime} C^{\bullet}(\bar{Z}, \mathbb{A}(k)) /{ }^{\prime} C_{E}^{\bullet}(\bar{Z}, \mathbb{A}(k)),
$$

where ${ }^{\prime} C_{E}^{\bullet}(\bar{Z}, \mathbb{A}(k)) \subset{ }^{\prime} C^{\bullet}(\bar{Z}, \mathbb{A}(k))$ is the subcomplex of chains supported on $E$.

Deligne homology ${ }^{\prime} H_{\mathcal{D}}^{\bullet}(Z, \mathbb{A}(k))$, as defined in [Jan88], is given by the cohomology of the complex

$$
\text { Cone }\left({ }^{\prime} C^{\bullet}(\bar{Z}, E, \mathbb{A}(k)) \oplus F^{k \prime} \Omega_{\bar{Z}_{\infty}}^{\bullet}\langle E\rangle(\bar{Z}) \stackrel{\epsilon-l}{\longrightarrow}{ }^{\prime} \Omega_{\bar{Z} \infty}^{\bullet}\langle E\rangle(\bar{Z})\right)[-1],
$$

where $\epsilon$ and $l$ are the natural maps of complexes. (The precise description of $\epsilon$ is given in [Jan88], and the required foundational material can be found in [Kin83].) Here we define $\Omega_{\bar{Z}}^{\bullet}\langle E\rangle=\Omega_{\bar{Z}}^{\bullet}(\log E)$ to be the de Rham complex of meromorphic forms on $\bar{Z}$, holomorphic on $U=\bar{Z}-E$, with at 


\section{KerR, J. D. Lewis ANd S. MÜLler-Stach}

most logarithmic poles along $E$. Also, $\Omega_{\bar{Z}^{\infty}}^{\bullet}\langle E\rangle=\Omega_{\bar{Z}}^{\bullet}\langle E\rangle \otimes_{\Omega_{\bar{Z}}^{\bullet}} \Omega_{\bar{Z}^{\infty}}$, and ' $\Omega_{\bar{Z}^{\infty}}^{n}\langle E\rangle$ is defined by the equivalent sheaves

$$
' \Omega_{\bar{Z}^{\infty}}^{n} /{ }^{\prime} \Omega_{\bar{Z}^{\infty}}^{n}(\text { on } E) \cong \mathcal{D}\left(\Omega_{\bar{Z}^{\infty}}^{-n}(\text { null } E)\right) \cong \bigoplus_{p+q=n} \Omega_{\bar{Z}}^{p+m}\langle E\rangle \otimes \mathcal{O}_{Z}{ }^{\prime} \Omega_{\bar{Z}}^{0, q} .
$$

There is thus a map of complexes ' $\Omega_{\bar{Z}_{\infty}}^{\bullet} \rightarrow{ }^{\prime} \Omega_{\bar{Z}_{\infty}}^{\bullet}\langle E\rangle$ which is surjective at each term; ' $\Omega_{\bar{Z}_{\infty}}^{\bullet}\langle E\rangle=$ $\Omega_{\bar{Z}}^{\bullet}\langle E\rangle \otimes_{\Omega_{\bar{Z}}^{\bullet}}{ }^{\prime} \Omega_{\bar{Z}^{\infty}}^{\bullet}$. The corresponding Hodge filtrations are $F^{i} \Omega_{\bar{Z}^{\infty}}^{\bullet}\langle E\rangle=\left\{F^{i} \Omega_{\bar{Z}}^{\bullet}\langle E\rangle\right\} \otimes_{\Omega_{\bar{Z}}^{\bullet}} \Omega_{\bar{Z}^{\infty}}^{\bullet}$, and $F^{i \prime} \Omega_{\bar{Z} \infty}^{\bullet}\langle E\rangle=\left\{F^{i+m} \Omega_{\bar{Z}}^{\bullet}\langle E\rangle\right\} \otimes_{\mathcal{O}_{\bar{Z}}}{ }^{\prime} \Omega_{\bar{Z}^{\infty}}^{0, \bullet}$. As is well known (see [Jan88]), there are filtered quasiisomorphisms

$$
\left(\Omega_{\bar{Z}}^{\bullet}\langle E\rangle, F^{i}\right) \hookrightarrow\left(\Omega_{\bar{Z}^{\infty}}^{\bullet}\langle E\rangle, F^{i}\right) \hookrightarrow\left({ }^{\bullet} \Omega_{\bar{Z}^{\infty}}\langle E\rangle[-2 m], F^{i-m}\right) .
$$

\section{Review of the classical situation $(n=0)$}

General references for this section are [EV88] and [Jan88]. For a codimension $p$ cycle $\mathcal{Z}$ on $X$, there is the localization sequence of mixed Hodge structures

$$
0 \rightarrow H^{2 p-1}(X, \mathbb{Z}(p)) \stackrel{\beta}{\rightarrow} H^{2 p-1}(X \backslash|\mathcal{Z}|, \mathbb{Z}(p)) \rightarrow H_{|\mathcal{Z}|}^{2 p}(X, \mathbb{Z}(p)) \rightarrow H^{2 p}(X, \mathbb{Z}(p)) .
$$

The map $\beta$ induces the isomorphism

$$
\frac{H^{2 p-1}(X, \mathbb{C})}{F^{p} H^{2 p-1}(X, \mathbb{C})} \simeq \frac{H^{2 p-1}(X \backslash|\mathcal{Z}|, \mathbb{C})}{F^{p} H^{2 p-1}(X \backslash|\mathcal{Z}|, \mathbb{C})}
$$

and hence the isomorphism

$$
J^{p}(X) \simeq \frac{H^{2 p-1}(X \backslash|\mathcal{Z}|, \mathbb{C})}{H^{2 p-1}(X, \mathbb{Z}(p))+F^{p} H^{2 p-1}(X \backslash|\mathcal{Z}|, \mathbb{C})} .
$$

Next, for $\mathcal{Z} \in Z_{\text {hom }}^{p}(X)$, the fundamental class $c_{\mathbb{Z}}(\mathcal{Z})$ is the image of a class $\widetilde{c_{\mathbb{Z}}(\mathcal{Z})} \in H^{2 p-1}(X \backslash$ $|\mathcal{Z}|, \mathbb{Z}(p))$, uniquely determined up to $\operatorname{Im}(\beta)$. Since $\widehat{c_{\mathbb{Z}}(\mathcal{Z})}$ defines a class in $H^{2 p-1}(X \backslash|\mathcal{Z}|, \mathbb{C}$ ) (still denoted by $\left.\widehat{c_{\mathbb{Z}}(\mathcal{Z})}\right)$, we end up with a corresponding class $\Psi_{p}(\mathcal{Z}) \in J^{p}(X)$ via the isomorphisms above. We use this as our initial definition, as follows.

Definition 3.1. The class $\Psi_{p}: Z_{\text {hom }}^{p}(X) \rightarrow J^{p}(X)$ is called the Abel-Jacobi map.

\subsection{Comparison to Carlson's Abel-Jacobi map}

The exact sequence (3.1) yields an extension

$$
0 \rightarrow H^{2 p-1}(X, \mathbb{Z}(p)) \rightarrow \mathbf{E} \rightarrow \mathbb{Z}(0) \rightarrow 0
$$

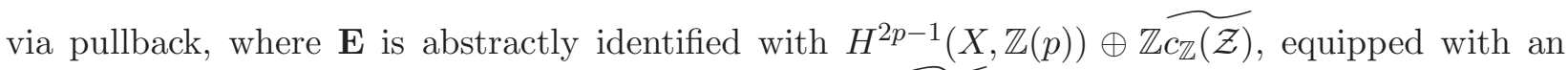
(integral) retraction $r_{\mathbb{Z}}: \mathbf{E} \rightarrow H^{2 p-1}(X, \mathbb{Z}(p))$ killing $\widetilde{c_{\mathbb{Z}}(\mathcal{Z})}$. This $r_{\mathbb{Z}}$ extends to a map $\mathbf{E}_{\mathbb{C}} \rightarrow$ $H^{2 p-1}(X, \mathbb{C})$.

If $\widetilde{c_{F}(\mathcal{Z})} \in E_{\mathbb{C}} \subseteq F^{p} H^{2 p-1}(X \backslash|\mathcal{Z}|, \mathbb{C})$ is another lift of the fundamental class of $\mathcal{Z}$ respecting the Hodge filtration, then Carlson's prescription [Car87] is $r_{\mathbb{Z}}\left(\widetilde{c_{F}(\mathcal{Z})}\right) \in J^{p}(X)$, its image under the retraction. Since $\widehat{c_{\mathbb{Z}}(\mathcal{Z})}$ and $\widehat{c_{F}(\mathcal{Z})}$ both lift the fundamental class, their difference lifts to an element $\xi \in H^{2 p-1}(X, \mathbb{C})$. We write this $\widetilde{c_{F}(\mathcal{Z})}=\widetilde{c_{\mathbb{Z}}(\mathcal{Z})}+\xi$; applying $r_{\mathbb{Z}}$ shows that $\xi=r_{\mathbb{Z}}\left(\widetilde{c_{F}(\mathcal{Z})}\right)$, and hence that $\widetilde{c_{F}(\mathcal{Z})}=\widetilde{c_{\mathbb{Z}}(\mathcal{Z})}+r_{\mathbb{Z}}\left(\widetilde{c_{F}(\mathcal{Z})}\right)$. Thus

$$
\widetilde{c_{\mathbb{Z}}(\mathcal{Z})}+r_{\mathbb{Z}}\left(\widetilde{c_{F}(\mathcal{Z})}\right) \equiv 0 \text { modulo } F^{p} H^{2 p-1}(X \backslash|\mathcal{Z}|, \mathbb{C}) .
$$

So we have the following proposition. 
Proposition 3.2. Carlson's Abel-Jacobi map is the same as $\Psi_{p}$, up to sign.

\subsection{Comparison to classical $A J$ map}

We proceed by comparing $\Psi_{p}$ with the Deligne cycle-class map in Proposition 3.3, and then identifying the latter with the classical $A J$ in Proposition 3.4.

Recall the following diagram of exact sequences.

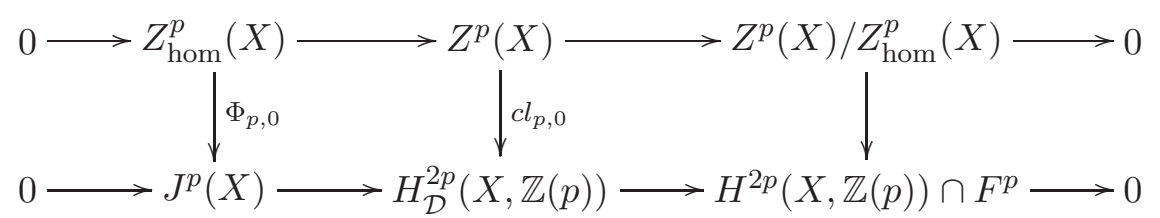

Proposition 3.3. We have $\Psi_{p}=\Phi_{p, 0}$.

Proof. See [EV88].

Finally, working with Deligne homology, we obtain the following result.

Proposition 3.4. The map $\Phi_{p, 0}$ coincides with the classical Abel-Jacobi map.

Proof. See [Jan88].

\section{A localization argument (first construction of $\boldsymbol{A J}$ )}

If $\mathcal{Z} \in X \times \square^{n}$ is irreducible and of codimension $p$, consider $V:=\overline{\pi_{*}(\mathcal{Z})} \subset X$, which we assume has dimension $m+n-p$. The $i$ th coordinate projections $\mathcal{Z} \rightarrow \square$ determine rational functions on $\mathcal{Z}$. Taking the norm of a symbol in Milnor $K$-theory, after passing to the relevant functions fields, reduces to the situation of rational functions $\left\{f_{1}, \ldots, f_{n}\right\}$ on $V$. Of course, when $n=1$, we are dealing with the usual norm $N: \mathbb{C}(\mathcal{Z})^{\times} \rightarrow \mathbb{C}(V)^{\times}$. On an open set $U_{V} \subset V$, we have elements $f_{j} \in H^{0}\left(U_{V}, \mathcal{O}_{U_{V}}^{\times}\right)=C H^{1}\left(U_{V}, 1\right)$. Let $U=X \backslash\left(V \backslash U_{V}\right)$. One has the following commutative diagram.

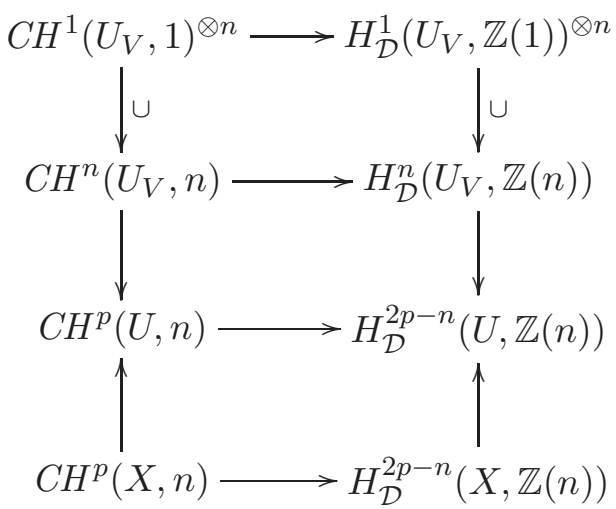

We come up with a formula for the regulator at the generic point, based on the cup-product formula in Deligne cohomology. We need the following basic lemma.

Lemma 4.1. Let $Y$ be a smooth quasiprojective variety, let $f: Y \rightarrow \mathbb{P}^{1}$ be a dominant morphism, and let $\eta \in E_{\bar{Y}}^{2 m-1}$. Then

$$
\int_{\bar{Y}} \frac{d f}{f} \wedge \eta=(2 \pi \mathrm{i}) \int_{f^{-1}[-\infty, 0]} \eta+d\left[T_{\log f}\right](\eta),
$$




\section{KerR, J. D. LeWis AND S. MÜLleR-StaCH}

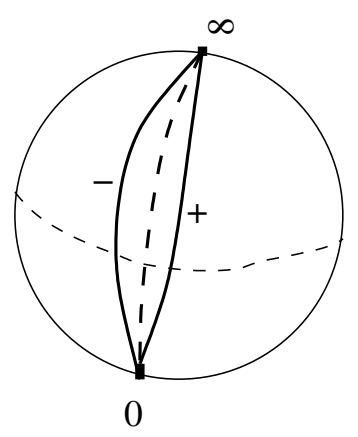

Figure 1. Slit sphere.

where

$$
T_{\log f}(\mu):=\int_{\bar{Y} \backslash f^{-1}[-\infty, 0]} \mu \log f
$$

and $[-\infty, 0]=\mathbb{R}^{-}$is oriented along $\mathbb{R}^{-}$so that $\partial[-\infty, 0]=\{0\}-\{\infty\}$.

Proof. One observes that $\log f$ is single-valued in $\bar{Y} \backslash f^{-1}[-\infty, 0]$, where $\log$ is the branch with $\arg \in(-\pi, \pi)$; so we have

$$
d((\log f) \eta)=\frac{d f}{f} \wedge \eta+(\log f) d \eta
$$

there. Let $B_{\epsilon}$ be an $\epsilon$-band angular sector neighborhood of $[-\infty, 0]=\mathbb{R}^{-}$in $\mathbb{P}^{1}$, with boundary $C_{ \pm}(\epsilon)$; see Figure 1 .

Put $D_{\epsilon}:=f^{-1}\left(B_{\epsilon}\right)$, and $L_{ \pm}(\epsilon)=\partial D_{\epsilon}=f^{-1}\left(C_{ \pm}(\epsilon)\right)$. Then

$$
\begin{aligned}
\int_{\bar{Y}} \frac{d f}{f} \wedge \eta & =\int_{\bar{Y} \backslash f^{-1}[-\infty, 0]} d((\log f) \eta)-\int_{\bar{Y}}(\log f) d \eta \\
& =\lim _{\epsilon \rightarrow 0^{+}} \int_{\bar{Y} \backslash D_{\epsilon}} d((\log f) \eta)+d\left[T_{\log f}\right](\eta) \\
& =-\lim _{\epsilon \rightarrow 0^{+}} \int_{L_{ \pm}(\epsilon)}(\log f) \eta+d\left[T_{\log f}\right](\eta) .
\end{aligned}
$$

But

$$
\lim _{\epsilon \rightarrow 0^{+}} L_{ \pm}(\epsilon)=f^{-1}[-\infty, 0]-f^{-1}[-\infty, 0]=0 .
$$

Since we pick up a period on log, we arrive at

$$
\int_{\bar{Y}} \frac{d f}{f} \wedge \eta=2 \pi \mathrm{i} \int_{f^{-1}[-\infty, 0]} \eta+d\left[T_{\log f}\right](\eta)
$$

as was to be shown.

We now want to consider the following setting. Let $V$ be an irreducible complex projective variety (in particular a component of the $V$ from before the lemma). Let $f_{1}, \ldots, f_{n} \in \mathbb{C}(V)^{\times}$, and put $D:=\bigcup_{j=1}^{n}\left|\left(f_{j}\right)\right| \cup V_{\text {sing }}, U_{V}:=V \backslash D$. Consider the pair $(\tilde{V}, \tilde{D})$, where $\tilde{V}$ is a smooth projective variety, $\tilde{D}$ a normal crossing divisor, and $\tilde{V} \backslash \tilde{D}=V \backslash D$. The Deligne (co)homology of $U_{V}$ can be computed in terms of the Deligne complex of the pair $(\tilde{V}, \tilde{D})$. We may assume that $f_{1}, \ldots, f_{n}: \tilde{V} \rightarrow \mathbb{P}^{1}$ are dominant morphisms. Notice that $\gamma_{j}:=f_{j}^{-1}[-\infty, 0]$ are (Borel-Moore) cycles on $U_{V}$. Let $v=\operatorname{dim} V(=m+n-p)$ and define

$$
T_{f_{j}}(\mu)=2 \pi \mathrm{i} \int_{\gamma_{j}} \mu, \quad \Omega_{f_{j}}(\mu)=\int_{\tilde{V}} \frac{d f_{j}}{f_{j}} \wedge \mu, \quad R_{f_{j}}(\mu)=\int_{\tilde{V}}\left(\log f_{j}\right) \mu .
$$




\section{The ABel-JACOBI MAP FOR Higher Chow Groups}

Before stating our next result, we recall the multiplication table [EV88], pertaining to

$$
\bigcup_{\alpha}: \mathbb{A}_{\mathcal{D}}(p) \otimes \mathbb{A}_{\mathcal{D}}(q) \rightarrow \mathbb{A}_{\mathcal{D}}(p+q)
$$

for any given $\alpha \in \mathbb{R}$. Here $\mathbb{A}_{\mathcal{D}}(p)$ is defined in [EV88] in terms of a cone complex, which is quasiisomorphic to the same labelled complex $\mathbb{A}_{\mathcal{D}}(p)$ that we introduced in $\S 2$.

\begin{tabular}{c|ccc} 
& $a_{q}$ & $f_{q}$ & $\omega_{q}$ \\
\hline$a_{p}$ & $a_{p} \cdot a_{q}$ & 0 & $(1-\alpha) \cdot a_{p} \cdot \omega_{q}$ \\
$f_{p}$ & 0 & $f_{p} \wedge f_{q}$ & $(-1)^{\operatorname{deg} f_{p}} \cdot \alpha \cdot f_{p} \cdot \omega_{q}$ \\
$\omega_{p}$ & $\alpha \cdot \omega_{p} \cdot a_{q}$ & $(1-\alpha) \cdot \omega_{p} \wedge f_{q}$ & 0
\end{tabular}

Evidently, up to homotopy, $\bigcup_{\alpha}$ is independent of $\alpha \in \mathbb{R}$ [EV88]. Now put $\bigcup=\bigcup_{\alpha=0}$. We have the following proposition.

Proposition 4.2. (i) For each $j$, the triple $\left(T_{f_{j}}, \Omega_{f_{j}}, R_{f_{j}}\right)$ defines a class

$$
\left\{\left(T_{f_{j}}, \Omega_{f_{j}}, R_{f_{j}}\right)\right\} \in H_{2 v-1}^{\mathcal{D}}\left(U_{V}, \mathbb{Z}(v-1)\right) \stackrel{\mathrm{PD}}{\simeq} H_{\mathcal{D}}^{1}\left(U_{V}, \mathbb{Z}(1)\right) .
$$

(ii) Via the cup product

$$
\bigcup_{j=1}^{n}\left\{\left(T_{f_{j}}, \Omega_{f_{j}}, R_{f_{j}}\right)\right\}=\left\{\left(T_{\mathbf{f}}, \Omega_{\mathbf{f}}, R_{\mathbf{f}}\right)\right\},
$$

where $\mathbf{f}=\left(f_{1}, \ldots, f_{n}\right)$,

$$
T_{\mathbf{f}}(\mu)=(2 \pi \mathrm{i})^{n} \int_{\left(f_{1} \times \cdots \times f_{n}\right)^{-1}[-\infty, 0]^{n}} \mu, \quad \Omega_{\mathbf{f}}(\mu)=\int_{V} \frac{d f_{1}}{f_{1}} \wedge \cdots \wedge \frac{d f_{n}}{f_{n}} \wedge \mu,
$$

and where

$$
\begin{aligned}
R_{\mathbf{f}}(\omega)=[ & \int_{V \backslash f_{1}^{-1}[-\infty, 0]}\left(\log f_{1}\right) \frac{d f_{2}}{f_{2}} \wedge \cdots \wedge \frac{d f_{n}}{f_{n}} \wedge \omega \\
& +(-2 \pi \mathrm{i}) \int_{f_{1}^{-1}[-\infty, 0] \backslash\left(f_{1} \times f_{2}\right)^{-1}[-\infty, 0]^{2}}\left(\log f_{2}\right) \frac{d f_{3}}{f_{3}} \wedge \cdots \wedge \frac{d f_{n}}{f_{n}} \wedge \omega+\cdots \\
& \left.+(-2 \pi \mathrm{i})^{n-1} \int_{\left(f_{1} \times \cdots \times f_{n-1}\right)^{-1}[-\infty, 0]^{n-1} \backslash\left(f_{1} \times \cdots \times f_{n}\right)^{-1}[-\infty, 0]^{n}}\left(\log f_{n}\right) \omega\right] .
\end{aligned}
$$

Proof. Part (i) is immediate from Lemma 4.1 and part (ii) uses the multiplication table above, the cone complex description of Deligne homology together with Poincaré duality, and induction on $n$.

Remark 4.3. As a consequence of part (ii) above, we have the Deligne homology relation

$$
\Omega_{\mathbf{f}}=T_{\mathbf{f}}+d\left[R_{\mathbf{f}}\right],
$$

as currents acting on forms that are compactly supported on $U_{V}$. Using induction, the proof of Lemma 4.1 can be generalized, which extends the above formula to act on forms on $V$, as follows.

Proposition 4.4. Consider (dominant) morphisms $f_{1}, \ldots, f_{n}$ from $V$ to $\mathbb{P}^{1}$, in general position and put

$$
R_{\partial \mathbf{f}}=\left.\sum_{j=1}^{n}(-1)^{j-1} R_{\left\{f_{1}, \ldots, \hat{f}_{j}, \ldots, f_{n}\right\}}\right|_{\left(f_{j}\right)} .
$$

Then

$$
\Omega_{\mathbf{f}}=T_{\mathbf{f}}+d\left[R_{\mathbf{f}}\right] \pm(2 \pi \mathrm{i}) R_{\partial \mathbf{f}}
$$




\section{KerR, J. D. Lewis AND S. MÜLler-StaCH}

Example 4.5. Suppose we are given a higher Chow cycle $\mathcal{Z}=\sum_{\alpha}\left(f_{\alpha}, V_{\alpha}\right) \in C H_{\text {hom }}^{p}(X, 1)$. Then by Hodge theory, we have $\sum_{\alpha} \Omega_{f_{\alpha}}=d S$, where by Poincaré duality $S \in F^{p}$ (which plays the role of $b_{0}$ in Remark 2.3) acts as the zero current on $F^{m-p+1} H^{2 m-2 p+2}(X)$. Note that $\gamma:=\sum_{\alpha} \gamma_{\alpha}$ bounds a chain $\zeta$, and thus $T_{\mathcal{Z}}:=\sum_{\alpha} T_{f_{\alpha}}=-2 \pi \mathrm{i} d \delta_{\zeta}$. Taking the coboundary (see $\S 2$ ), viz.

$$
\delta\left(-\delta_{\zeta}, S, 0\right)=\left(-T_{\mathcal{Z}},-\sum_{\alpha} \Omega_{\alpha},-2 \pi \mathrm{i} \delta_{\zeta}-S\right),
$$

this leads us to

$$
\left(T_{\mathcal{Z}}, \sum_{\alpha} \Omega_{f_{\alpha}}, \sum_{\alpha} R_{f_{\alpha}}\right) \sim\left(0,0, \sum_{\alpha} R_{f_{\alpha}}-2 \pi \mathrm{i} \delta_{\zeta}-S\right)
$$

in Deligne homology. By applying Poincaré duality, this leads us to Levine's formula [Lev88] for the regulator on $K_{1}$, induced by

$$
\begin{gathered}
\omega \in F^{m-p+1} \Omega_{X^{\infty}}^{2 m-2 p+2}(X) \\
\mapsto \frac{1}{(2 \pi \mathrm{i})^{m-p+1}}\left(\sum_{\alpha} \int_{V_{\alpha} \backslash f_{\alpha}^{-1}[-\infty, 0]}\left(\log f_{\alpha}\right) \omega-2 \pi \mathrm{i} \int_{\zeta} \omega\right) .
\end{gathered}
$$

[Note: In Levine's formula, the $-2 \pi \mathrm{i} \int_{\zeta} \omega$ is replaced by $+2 \pi \mathrm{i} \int_{\zeta} \omega$. This is because he is using the branch of the logarithm with imaginary part $\in(0,2 \pi)$. Also, we have used the homological version of the Tate twist, which includes the factor $(2 \pi \mathrm{i})^{m}$.]

\section{The map of complexes (second construction of $A J$ )}

We first describe the notation we shall use, which is a bit more involved than that of the preceding section (there are also slight differences).

\subsection{Notation for currents}

Let $\mathcal{X}$ be a quasiprojective variety of complex dimension $m, Y \subset \mathcal{X}$ an oriented analytic subset of real codimension $k$, and $\Omega \in \Gamma\left(\Omega_{\mathcal{X}}^{\ell}(\log D)\right)$ where $D \subset \mathcal{X}$ is any divisor. Associate to any given meromorphic function $f \in \mathbb{C}(\mathcal{X})$ the $(2 m-1)$-chain $T_{f}:=\overline{f^{-1}\left(\mathbb{R}^{-}\right)}$oriented so that $\partial T_{f}=(f)=$ $\left|(f)_{0}\right|-\left|(f)_{\infty}\right|$. Now define a current $(\log f) \Omega \cdot \delta_{Y} \in F^{k^{\prime}} \mathcal{D}^{\ell+k}(\mathcal{X})$ by

$$
\int_{\mathcal{X}}(\log f) \Omega \cdot \delta_{Y} \wedge \omega:=\lim _{\epsilon \rightarrow 0} \int_{Y \backslash \mathcal{N}_{\epsilon}\left(D \cup T_{f}\right)}(\log f) \Omega \wedge \iota_{Y}^{*} \omega
$$

provided the limit exists for every $C^{\infty}$-form $\omega \in \Gamma\left(\Omega_{\mathcal{X}^{\infty}}^{2 m-\ell-k}\right)$ compactly supported away from the boundary of $\mathcal{X}$. (The $F^{k}$ means that all $\omega \in F^{m-k+1}$ are annihilated.)

Remark. To reiterate, on the right-hand side, ' $\log f$ ' is always taken to have imaginary part $\epsilon$ $(-\pi, \pi)$.

Recall that to any $i$-current $\mathcal{K}$ is associated an $(i+1)$-current $\mathrm{d}[\mathcal{K}]$ :

$$
\int_{\mathcal{X}} \mathrm{d}[\mathcal{K}] \wedge \omega=(-1)^{i+1} \int_{\mathcal{X}} \mathcal{K} \wedge \mathrm{d} \omega
$$

So for example $\mathrm{d}[\log f]=\operatorname{dlog} f-2 \pi \mathrm{i} \delta_{T_{f}}$, and $\mathrm{d}[\mathrm{d} \log f]=2 \pi \mathrm{i} \delta_{(f)}$.

\subsection{Higher Chow groups}

We shall use the notation

$$
\square^{n}:=\left(\mathbb{P}_{\mathbb{C}}^{1} \backslash\{1\}\right)^{n} \quad \text { with coordinates }\left(z_{1}, \ldots, z_{n}\right)
$$


for affine $n$-space, with subsets

$$
\begin{aligned}
\partial \square^{n} & :=\bigcup_{i=1}^{n}\left\{\left(z_{1}, \ldots, z_{n}\right) \in \square^{n} \mid z_{i} \in\{0, \infty\}\right\} \\
& =\text { faces of } \square^{n}
\end{aligned}
$$

and

$$
\begin{aligned}
\partial^{k} \square^{n} & :=\bigcup_{i_{1}<\cdots<i_{k}}\left\{\left(z_{1}, \ldots, z_{n}\right) \in \square^{n} \mid z_{i_{1}}, \ldots, z_{i_{k}} \in\{0, \infty\}\right\} \\
& =\text { codimension- } k \text { subfaces. }
\end{aligned}
$$

Also let

$$
\mathcal{N}_{\epsilon}\left(\partial \square^{n}\right):=\bigcup_{i=1}^{\infty}\left\{\left(z_{1}, \ldots, z_{n}\right) \in \square^{n}|| z_{i} \mid<\epsilon \text { or }\left|z_{i}\right|>\frac{1}{\epsilon}\right\}
$$

and $\square_{\epsilon}^{n}:=\square^{n} \backslash \mathcal{N}_{\epsilon}\left(\partial \square^{n}\right)$.

Let $X$ be a complex projective variety of dimension $m$, and define subgroups of algebraic cycles on $X \times \square^{n}$,

$$
Z^{p}\left(X \times \square^{n}\right) \supseteq c^{p}(X, n) \supseteq d^{p}(X, n),
$$

generated (respectively) by those subvarieties intersecting all subfaces $X \times \partial^{k} \square^{n}$ properly, and (among those) by subvarieties pulled back from $X \times$ face by a coordinate projection. Set $Z^{p}(X, n):=$ $c^{p}(X, n) / d^{p}(X, n)$; writing $\rho_{i}^{0}, \rho_{i}^{\infty}$ for the inclusions of the $i$ th faces, define Bloch's differential

$$
\partial_{\mathcal{B}}:=\sum_{i=1}^{n}(-1)^{i-1}\left(\rho_{i}^{\infty *}-\rho_{i}^{0 *}\right): Z^{p}(X, n) \rightarrow Z^{p}(X, n-1) .
$$

Since $\partial_{\mathcal{B}} \circ \partial_{\mathcal{B}}=0$ this gives a complex, with $C H^{p}(X, n)$ as homology groups; only for our purposes cohomological indexing is better and we shall write

$$
C H^{p}(X, n):=H^{-n}\left\{Z^{p}(X,-\bullet)\right\} .
$$

\subsection{Currents on $\square^{n}$}

Set

$$
\begin{aligned}
\Omega^{n}=\Omega\left(z_{1}, \ldots, z_{n}\right): & =\operatorname{dlog} z_{1} \wedge \cdots \wedge \operatorname{dlog} z_{n} \in F^{n \prime} \mathcal{D}^{n}\left(\square^{n}\right), \quad \text { holomorphic } n \text {-current } \\
T^{n}: & =T_{z_{1}} \cap \cdots \cap T_{z_{n}} \in \mathcal{C}_{n}\left(\square^{n}\right) \text { topological } n \text {-chain } \\
R^{n}= & R\left(z_{1}, \ldots, z_{n}\right) \\
:= & \log z_{1} \operatorname{dlog} z_{2} \wedge \cdots \wedge \operatorname{dlog} z_{n} \\
& +( \pm 2 \pi \mathrm{i}) \log z_{2} \operatorname{dlog} z_{3} \wedge \cdots \wedge \operatorname{dlog} z_{n} \cdot \delta_{T_{z_{1}}}+\cdots \\
& +( \pm 2 \pi \mathrm{i})^{n-1} \log z_{n} \cdot \delta_{T_{z_{1}} \cap \cdots \cap T_{z_{n-1}}} \in{ }^{\prime} \mathcal{D}^{n-1}\left(\square^{n}\right),
\end{aligned}
$$

where ' \pm ' means $(-1)^{n-1}$. For example, $R^{1}=\log z$ and $R^{2}=\log z_{1} \operatorname{dlog} z_{2}-2 \pi \mathrm{i} \log z_{2} \cdot \delta_{T_{z_{1}}}$. One may view these also as currents on $X \times \square^{n}$ by pullback.

From above one has for $n=1, \mathrm{~d}\left[R^{1}\right]=\Omega^{1}-2 \pi \mathrm{i} \cdot \delta_{T^{1}}$, which generalizes to $n>1$ via

$$
\mathrm{d}\left[R^{n}\right]=\Omega^{n}-(2 \pi \mathrm{i})^{n} \delta_{T^{n}}-(2 \pi \mathrm{i}) \sum_{i=1}^{n}(-1)^{i} R\left(z_{1}, \ldots, \hat{z}_{i}, \ldots, z_{n}\right) \cdot \delta_{\left(z_{i}\right)} .
$$

Moreover,

$$
\mathrm{d}\left[\Omega^{n}\right]=2 \pi \mathrm{i} \sum_{i=1}^{n}(-1)^{i} \Omega\left(z_{1}, \ldots, \hat{z}_{i}, \ldots, z_{n}\right) \cdot \delta_{\left(z_{i}\right)}
$$




\section{KerR, J. D. Lewis and S. MÜller-Stach}

and

$$
\partial T^{n}=\sum_{i=1}^{n}(-1)^{i}\left(\rho_{i *}^{0} T^{n-1}-\rho_{i}^{\infty} T^{n-1}\right) .
$$

\subsection{Currents on $X$}

To produce these we must first specify a subcomplex of $Z^{p}(X, \bullet)$ consisting of elements in good position with respect to certain real subsets of $X \times \square^{n}$. Denote by $T_{z_{i}}^{\circ}=z_{i}^{-1}\left(\mathbb{R}^{-}\right)=z_{i}^{-1}((-\infty, 0))$ the interior of the real $(2 n-1)$-chain $T_{z_{i}}=z_{i}^{-1}([-\infty, 0])$ on $\square^{n}$. Let $c_{\mathbb{R}}^{p}(X, n)$ consist of all $\mathcal{Z} \in c^{p}(X, n)$ intersecting

$$
X \times\left(T_{z_{1}} \cap \cdots \cap T_{z_{j}}\right) \quad \text { and } \quad X \times\left\{\overline{\left(T_{z_{1}}^{\circ} \cap \cdots \cap T_{z_{j}}^{\circ}\right) \cap \partial^{k} \square^{n}}\right\}
$$

properly (for all $1 \leqslant j \leqslant n, 1 \leqslant k<n$ ), and $d_{\mathbb{R}}^{p}(X, n):=c_{\mathbb{R}}^{p}(X, n) \cap d^{p}(X, n)$. Then $Z_{\mathbb{R}}^{p}(X, \bullet):=$ $c_{\mathbb{R}}^{p}(X, \bullet) / d_{\mathbb{R}}^{p}(X, \bullet)$ is a complex under $\partial_{\mathcal{B}}$. A moving technique based on unpublished notes of Bloch and worked out and extended by Levine in [Lev03, § 1.3.4] and [Lev98, § 3.5.12] shows that this is quasiisomorphic to the Bloch complex, as follows.

Moving By tRANSLATION LEMma. We have $Z_{\mathbb{R}}^{p}(X, \bullet) \stackrel{\simeq}{\rightarrow} Z^{p}(X, \bullet)$.

The proof consists of showing that any cycle is equivalent to one in general position after a generic complex affine translation in $\Delta^{n}$. Such cycles are obviously contained in $Z_{\mathbb{R}}^{p}(X, \bullet)$. Note that the arguments given by Levine use simplicial coordinates. But the quasiisomorphism between simplicial and cubical coordinate systems can be applied here. Alternatively one could restate the moving by translation lemma in cubical coordinates and prove it there. Let us offer an indication of what the 'move' is. Any $\mathcal{Z} \in Z^{p}(X, n)$ is defined over some $k \subseteq \mathbb{C}$ finitely generated $/ \overline{\mathbb{Q}}$. Now consider $\alpha_{1}, \ldots, \alpha_{n} \in \mathbb{C}^{*}$ such that $\operatorname{trdeg}\left(k\left(\alpha_{1}, \ldots, \alpha_{n}\right) / k\right)=n$, and let $\tau=\left(\alpha_{1}, \ldots, \alpha_{n}\right)$ act by multiplication on the coordinates $\left(z_{1}, \ldots, z_{n}\right)$ of $\mathcal{Z}$ in $\square^{n}$.

Proposition. Under these conditions, $\tau \cdot \mathcal{Z} \in Z_{\mathbb{R}}^{p}(X, n)$.

(Of course, $\tau \cdot \mathcal{Z}$ is no longer defined over $k$.)

With some work, this proposition can be used to produce a map of complexes $T: Z^{p}(X / k, \bullet) \rightarrow$ $Z_{\mathbb{R}}^{p}(X, \bullet)$ together with a homotopy $\mathcal{H}: Z^{p}(X / k, \bullet) \rightarrow Z^{p}(X, \bullet+1)$ respecting $Z_{\mathbb{R}}^{p}$ and subsets of $X$, which satisfies $T(\mathcal{Z})-\mathcal{Z}=\partial_{\mathcal{B}} \mathcal{H}(\mathcal{Z})+\mathcal{H}\left(\partial_{\mathcal{B}} \mathcal{Z}\right)$. The lemma follows.

Associated to $\mathcal{Z} \in Z^{p}(X, n)$ one now produces $\Omega_{\mathcal{Z}} \in F^{p \prime} \mathcal{D}^{2 p-n}(X), R_{\mathcal{Z}} \in{ }^{\prime} \mathcal{D}^{2 p-n-1}(X)$ by the formulas

$$
\int_{X}\left\{\begin{array}{l}
\Omega_{\mathcal{Z}} \\
R_{\mathcal{Z}}
\end{array}\right\} \wedge \omega:=\lim _{\epsilon \rightarrow 0} \sum_{j} n_{j} \int_{\mathcal{Z}_{\epsilon}^{j}} \pi_{\square}^{\mathcal{Z}_{j *}}\left\{\begin{array}{l}
\Omega^{n} \\
R^{n}
\end{array}\right\} \wedge \pi_{X}^{\mathcal{Z}_{j}} \omega,
$$

where $z=\sum n_{j} \mathcal{Z}_{j}\left(\mathcal{Z}_{j}\right.$ irreducible), $\mathcal{Z}_{j}^{\epsilon}:=\mathcal{Z}_{j} \cap\left(X \times \square_{\epsilon}^{n}\right)$, and $\omega$ is any $C^{\infty}$-form of the right degree. These currents are zero if $\mathcal{Z} \in d^{p}(X, n)$.

Remark. (i) That the limit on the right-hand side always converges follows from an elementary analytic argument, in which the proper intersection condition on each $\mathcal{Z}_{j}$ plays a crucial role (e.g., see [Ker03] for the proof for $\Omega_{\mathcal{Z}}$ ).

(ii) An appealing alternative form of the definition, e.g. for $R_{\mathcal{Z}}$, is $R_{\mathcal{Z}}=\sum n_{j} \pi_{X}^{\mathcal{Z}_{j}} \pi_{\square}^{\mathcal{Z}_{j *}} R^{n}$. The push-forward $\pi_{X *}^{\mathcal{Z}_{j}}$ should be regarded as involving integration for those $j$ for which $\mathcal{Z}_{j}$ has fibers of $\operatorname{dim} \geqslant 1$ over $X$. Note in particular that the numbers $\operatorname{codim}_{X}\left\{\operatorname{supp} \pi_{X}\left(\mathcal{Z}_{j}\right)\right\}$ are not in general all the same.

Finally set $T_{\mathcal{Z}}:=\sum_{j} n_{j} \cdot \pi_{X}\left\{\mathcal{Z}_{j} \cap\left(X \times T^{n}\right)\right\} \in \mathcal{C}_{2 m-2 p+n}(X)$. The relations (5.2)-(5.4) give rise to formulas

$$
\partial T_{\mathcal{Z}}=T_{\partial_{\mathcal{B}} \mathcal{Z}}, \quad \mathrm{d}\left[\Omega_{\mathcal{Z}}\right]=2 \pi \mathrm{i} \Omega_{\partial_{\mathcal{B}} \mathcal{Z}}, \quad \mathrm{d}\left[R_{\mathcal{Z}}\right]=\Omega_{\mathcal{Z}}-(2 \pi \mathrm{i})^{n} \delta_{T_{\mathcal{Z}}}-2 \pi \mathrm{i} R_{\partial_{\mathcal{B}} \mathcal{Z}}
$$




\subsection{The map of complexes}

Define a complex of cochains for the Deligne homology of $X$,

$$
\begin{aligned}
\mathcal{C}_{\mathcal{D}}^{\bullet-2 m}(X, \mathbb{Z}(p-m)): & =\text { Cone }\left\{\begin{array}{c}
\mathcal{C}_{2 m-\bullet}(X, \mathbb{Z}(p)) \\
\oplus \\
F^{\prime \prime} \mathcal{D}^{\bullet}(X)
\end{array} \rightarrow{ }^{\prime} \mathcal{D}^{\bullet}(X)\right\}[-1](-m) \\
& =\left\{\mathcal{C}_{2 m-\bullet}(X, \mathbb{Z}(p)) \oplus F^{p \prime} \mathcal{D}^{\bullet}(X) \oplus^{\prime} \mathcal{D}^{\bullet-1}(X)\right\}(-m)
\end{aligned}
$$

with differential $D$ taking $(a, b, c) \mapsto\left(-\partial a,-\mathrm{d}[b], \mathrm{d}[c]-b+\delta_{a}\right)$. Then according to the formulas (5.5), sending

$$
\mathcal{Z} \mapsto \frac{(-2 \pi \mathrm{i})^{p-n}}{(2 \pi \mathrm{i})^{m}}\left((2 \pi \mathrm{i})^{n} T_{\mathcal{Z}}, \Omega_{\mathcal{Z}}, R_{\mathcal{Z}}\right)=: \mathcal{R}_{X}(\mathcal{Z})
$$

produces a map of complexes

$$
\mathcal{R}_{X}: Z_{\mathbb{R}}^{p}(X,-\bullet) \rightarrow \mathcal{C}_{\mathcal{D}}^{2 p-2 m+\bullet}(X, \mathbb{Z}(p-m)) ;
$$

that is, $D \mathcal{R}_{X}(\mathcal{Z})=\mathcal{R}_{X}\left(\partial_{\mathcal{B}} \mathcal{Z}\right)$. (Note: $\Omega_{\mathcal{Z}}=0$ if $p>m$ or $p<n$.) According to the moving lemma we may replace $Z_{\mathbb{R}}^{p}(X,-\bullet)$ by $Z^{p}(X,-\bullet)$ with the caveat that the map is in the derived category. This induces the desired map

$$
A J: C H^{p}(X, n) \rightarrow H_{2 m-2 p+n}^{\mathcal{D}}(X, \mathbb{Z}(m-p)) \underset{\mathrm{PD}}{\stackrel{\cong}{\leftrightarrows}} H_{\mathcal{D}}^{2 p-n}(X, \mathbb{Z}(p))
$$

If $\partial_{\mathcal{B}} \mathcal{Z}=0$ then $\mathcal{Z}$ represents a class $[\mathcal{Z}] \in C H^{p}(X, n)$, and we write $A J[\mathcal{Z}]$ or $\Phi_{p, n}(\mathcal{Z})$ for the class $\left[\mathcal{R}_{X}(\mathcal{Z})\right]$.

Remark. (i) $A J$ is in fact a ring homomorphism; that is, if $[\mathcal{W}] \in C H^{p}(X, \ell)$ and $[\mathcal{Y}] \in C H^{q}(X, n)$ then $[\mathcal{W} \times \mathcal{Y}] \in C H^{p+q}(X, \ell+n)$ and

$$
\left[\mathcal{R}_{X}(\mathcal{W})\right] \cup\left[\mathcal{R}_{X}(\mathcal{Y})\right]=\left[\mathcal{R}_{X}(\mathcal{W} \times \mathcal{Y})\right]
$$

under the cup product in Deligne (co)homology. The class on the left-hand side is given (modulo factors of $2 \pi \mathrm{i}$ ) by

$$
\left((2 \pi \mathrm{i})^{\ell+n} T_{\mathcal{W}} \cap T_{\mathcal{Y}}, \Omega_{\mathcal{W}} \wedge \Omega_{\mathcal{Y}},(-1)^{\ell}(2 \pi \mathrm{i})^{\ell} \delta_{T_{\mathcal{W}}} \cdot R_{\mathcal{Y}}+R_{\mathcal{W}} \wedge \Omega_{\mathcal{Y}}\right)
$$

that this equals $\left((2 \pi \mathrm{i})^{\ell+n} T_{\mathcal{W} \times \mathcal{Y}}, \Omega_{\mathcal{W} \times \mathcal{Y}}, R_{\mathcal{W} \times \mathcal{Y}}\right)$ is implied by the formula

$$
\begin{aligned}
R\left(w_{1}, \ldots, w_{\ell} ; y_{1}, \ldots, y_{n}\right)= & (-1)^{\ell}(2 \pi i)^{\ell} \delta_{T\left(w_{1}, \ldots, w_{\ell}\right)} \cdot R\left(y_{1}, \ldots, y_{n}\right) \\
& +R\left(w_{1}, \ldots, w_{\ell}\right) \wedge \Omega\left(y_{1}, \ldots, y_{n}\right)
\end{aligned}
$$

on $\square^{\ell+n}=\square^{\ell} \times \square^{n}$ (with coordinates $w_{1}, \ldots, w_{\ell} ; y_{1}, \ldots, y_{n}$ ).

(ii) The projection of this $A J$ map to the real Deligne cohomology, i.e. the composition

$$
C H^{p}(X, n) \underset{A J}{\longrightarrow} H_{\mathcal{D}}^{2 p-n}(X, \mathbb{Z}(p)) \underset{\pi_{\mathbb{R}}}{\longrightarrow} H_{\mathcal{D}}^{2 p-n}(X, \mathbb{R}(n)),
$$

agrees exactly with the regulator map defined by Goncharov in [Gon95] (see [Ker03]).

(iii) On the other hand, the $A J$ map defined in [Gon95] was not correct. Indeed, the formula there is given by a sum of the real regulator with a membrane integral. If one adopted the $A J$ map defined in [Gon95], then the formula in Example 4.5 above would involve replacing $\log f_{\alpha}$ by $\log \left|f_{\alpha}\right|$, resulting in a current that would not descend to the level of cohomology.

\subsection{Passage to ordinary cohomology}

Now let $n \geqslant 1$ and $\mathcal{Z}$ be a higher Chow cycle: $\partial_{\mathcal{B}} \mathcal{Z}=0$. Then $\mathrm{d}\left[\Omega_{\mathcal{Z}}\right]=0, \partial T_{\mathcal{Z}}=0$, and $\mathrm{d}\left[R_{\mathcal{Z}}\right]=$ $\Omega_{\mathcal{Z}}-(2 \pi \mathrm{i})^{n} T_{\mathcal{Z}}$ imply that $\left[\Omega_{\mathcal{Z}}\right]=(2 \pi \mathrm{i})^{n} T_{\mathcal{Z}}$ in $H^{2 p-n}(X, \mathbb{C})$. Multiplying by $(2 \pi \mathrm{i})^{p-n}$, we get a 


\section{KerR, J. D. Lewis AND S. MÜlleR-StaCH}

class in

$$
F^{p} H^{2 p-n}(X, \mathbb{C}) \cap H^{2 p-n}(X, \mathbb{Z}(p)) .
$$

This is the closest thing we get to a 'fundamental class' for $\mathcal{Z}$; when $T_{\mathcal{Z}} \sim 0$ we say $[\mathcal{Z}] \in$ $C H_{\text {hom }}^{p}(X, n)$.

For $X$ projective (and $n \geqslant 1),(*)$ is a torsion group. Consequently, the class

$$
\left[\Omega_{\mathcal{Z}}\right] \in F^{p} H^{2 p-n}(X, \mathbb{C})
$$

is zero while $\left[T_{\mathcal{Z}}\right] \in H^{2 p-n}(X, \mathbb{Z})$ is at worst torsion.

To proceed further we must have $T_{\mathcal{Z}} \sim 0$. So, in general, we must either (a) pass to rational coefficients (to render $(*)$ zero) or (b) assume the slight restriction $[\mathcal{Z}] \in C H_{\text {hom }}^{p}(X, n)$. While we have chosen (b), we emphasize that what follows (for the remainder of the paper) works essentially verbatim with $\mathbb{Z}$ replaced everywhere by $\mathbb{Q}$ (instead of this assumption). Moreover, if $X$ is such that $H^{2 p-n}(X, \mathbb{Z})$ has no torsion, $C H^{p}(X, n)=C H_{\text {hom }}^{p}(X, n)$ and no such choice is necessary. (Trivial example: $X=$ pt.)

Assuming, then, that $\mathcal{Z}$ (i.e. $T_{\mathcal{Z}}$ ) is nullhomologous, there exist 'primitives' $\Xi \in F^{p \prime} \mathcal{D}^{2 p-n-1}(X)$, $\zeta \in \mathcal{C}_{2 m-2 p+n+1}(X, \mathbb{Z})$ such that $\mathrm{d}[\Xi]=\Omega_{\mathcal{Z}},(-1)^{n} d\left[\delta_{\zeta}\right]=\delta_{\partial \zeta}=T_{\mathcal{Z}}$ (or, strictly speaking, $\delta_{T_{\mathcal{Z}}}$ ). Here $\zeta$ is called a 'membrane'.

We may now modify $\mathcal{R}_{X}(\mathcal{Z})$ by a coboundary, to get

$$
\left((2 \pi \mathrm{i})^{n} T_{\mathcal{Z}}, \Omega_{\mathcal{Z}}, R_{\mathcal{Z}}\right)+D\left((-2 \pi \mathrm{i})^{n} \zeta, \Xi, 0\right)=\left(0,0, R_{\mathcal{Z}}-\Xi+(-2 \pi \mathrm{i})^{n} \delta_{\zeta}=: R_{\mathcal{Z}}^{\prime \prime}\right) .
$$

Now $\Xi$ and $(-2 \pi \mathrm{i})^{p} \zeta$ are ambiguous by $F^{p} H^{2 p-n-1}(X, \mathbb{C})$ and $H^{2 p-n-1}(X, \mathbb{Z}(p))$, respectively, and so we have a well-defined class

$$
(-2 \pi \mathrm{i})^{p-n}\left[R_{\mathcal{Z}}^{\prime \prime}\right] \in \frac{H^{2 p-n-1}(X, \mathbb{C})}{F^{p} H^{2 p-n-1}(X, \mathbb{C})+H^{2 p-n-1}(X, \mathbb{Z}(p))}
$$

reflecting the isomorphism of the latter group with $H_{\mathcal{D}}^{2 p-n}(X, \mathbb{Z}(p))$ for $n \geqslant 1$ and $X$ projective. [Note: $(2 \pi \mathrm{i})^{m}$ has already disappeared in the $\mathrm{PD} \cong$.] Since this quotient is equivalent to

$$
\left\{F^{m-p+1} H^{2 m-2 p+n+1}(X, \mathbb{C})\right\}^{\vee} / \operatorname{im}\left\{H_{2 m-2 p+n+1}(X, \mathbb{Z}(p))\right\},
$$

this class (and thus $A J[\mathcal{Z}]$ ) is computed by the functional

$$
\frac{1}{(-2 \pi \mathrm{i})^{n-p}} \int_{X} R_{\mathcal{Z}}^{\prime} \wedge(\cdot) \text { modulo periods }(2 \pi \mathrm{i})^{p} \int_{\gamma}(\cdot),
$$

where $R_{\mathcal{Z}}^{\prime}:=R_{\mathcal{Z}}+(2 \pi \mathrm{i})^{n} \delta_{\zeta}$ and we may drop the $\Xi$-term, again thanks to Hodge-type considerations (see Remark 2.3).

Example. One easily recovers Levine's formula for $C H^{p}(X, 1)$ from this approach, writing $R^{1}=\log z$ and

$$
R_{\mathcal{Z}}=\sum_{\alpha} \pi_{X}^{\left(\mathcal{Z}_{\alpha}, f_{\alpha}\right)}{ }_{*} \pi_{\square}^{\left(\mathcal{Z}_{\alpha}, f_{\alpha}\right)_{*}}(\log z)=\sum_{\alpha} \log f_{\alpha} \cdot \delta_{\mathcal{Z}_{\alpha}}
$$

\subsection{A further simplification for $n \geqslant p$ or $p>m$}

For $p$ in this range we may clean up the above functional considerably; since then $F^{p} H^{2 p-n-1}(X, \mathbb{C})$ $=0$ and $F^{m-p+1} \Omega_{X^{\infty}}^{2 m-2 p+n+1}(X)=F^{0} \Omega_{X^{\infty}}^{2 m-2 p+n+1}(X)$, we may as well evaluate it on integral classes, that is, Poincaré duals of topological $(2 p-n-1)$-cycles $\xi$. The 'periods' are then all in $\mathbb{Z}(p)$, as is the contribution from the term $(2 \pi i)^{n} \delta_{\zeta}$ in $R_{\mathcal{Z}}^{\prime}$. One may therefore regard the functional

$$
\frac{1}{(-2 \pi \mathrm{i})^{n-p}} \int_{(\cdot)} R_{\mathcal{Z}} \in \operatorname{Hom}\left(H_{2 p-n-1}(X, \mathbb{Z}), \mathbb{C} / \mathbb{Z}(p)\right)
$$

as representing $A J[\mathcal{Z}] \in H^{2 p-n-1}(X, \mathbb{C} / \mathbb{Z}(p))$. 


\section{The ABEL-JACOBI MAP FOR HIGHER CHOW GROUPS}

Example. In the very simple (but interesting) series of examples $C H^{p}$ (pt., $2 p-1$ ), $R_{\mathcal{Z}}$ is simply the number $\int_{\mathcal{Z}} R^{2 p-1}$, and the resulting classes

$$
A J[\mathcal{Z}]=\frac{1}{(-2 \pi \mathrm{i})^{p-1}} \int_{\mathcal{Z}} R^{2 p-1} \in \mathbb{C} / \mathbb{Z}(p) \cong H_{\mathcal{D}}^{1}(\text { pt. }, \mathbb{Z}(p))
$$

are related to Goncharov's Chow $p$-logarithm [Gon95]. Here we just want to point out (for $p=2$ ) how to construct classes in $C H^{2}$ (pt., 3 ) with torsion and nontorsion $A J$ images. Note that for $p=2$ the above formula simplifies to

$$
-A J[\mathcal{Z}]=\int_{\mathcal{Z} \cap T_{z_{1}}} \log z_{2} \operatorname{dlog} z_{3}+2 \pi i \sum_{p \in \mathcal{Z} \cap T_{z_{1}} \cap T_{z_{2}}} \log z_{3}(p),
$$

since one integral vanishes for type reasons. Consider $a, b \in \mathbb{C}^{*} \backslash\{1\}$ and introduce

$$
\begin{aligned}
& V(a)=\left\{(1-a / t, 1-t, t) \mid t \in \mathbb{P}^{1}\right\} \cap \square^{3}, \\
& W(b)=\left\{(1-b / t, t, 1-t) \mid t \in \mathbb{P}^{1}\right\} \cap \square^{3} .
\end{aligned}
$$

Then one easily shows that

$$
\partial V(a)=(1-a, a), \quad \partial W(b)=(b, 1-b) .
$$

Hence $\xi_{a}:=V(a)-W(1-a)$ defines a class in $C H^{2}($ pt., 3$)$.

The value $\Phi_{2,3}\left(\xi_{a}\right) \in \mathbb{C} / \mathbb{Z}(2)$ is not hard to compute. Noting that $\xi_{a} \in c_{\mathbb{R}}^{2}$ (pt., 3$)$ for $1-a \notin \mathbb{R}^{-}$, one finds that

$$
\Phi_{2,3}\left(\xi_{a}\right)=\mathrm{Li}_{2}(a)+\mathrm{Li}_{2}(1-a)+\log a \log (1-a),
$$

where $\mathrm{Li}_{2}$ is the dilogarithm, and log is the principal branch. By Beilinson's rigidity [Bei85], this value does not depend on $a$. Hence

$$
\Phi_{2,3}\left(\xi_{a}\right)=\lim _{a \rightarrow 0} \Phi_{2,3}\left(\xi_{a}\right)=\sum_{n=1}^{\infty} \frac{1}{n^{2}}=\frac{\pi^{2}}{6} \in \mathbb{C} / \mathbb{Z}(2)
$$

is a torsion class.

Next, let $D_{2}$ be the Bloch-Wigner function, $\mathcal{B}_{2}(\mathbb{C})$ be the Bloch group, and st : $\mathcal{B}_{2}(\mathbb{C}) \rightarrow$ $\mathbb{C}^{*} \wedge \mathcal{Z} \mathbb{C}^{*}$ the standard map $\{a\}_{2} \mapsto(1-a) \wedge a$. Finally set $\rho(a)=\operatorname{Alt}_{3}(V(a))$. The following proposition is proved in [Ker03, $\S 3.1 .2]$.

Proposition. Given any element $\sum m_{j}\left\{a_{j}\right\}_{2} \in \operatorname{ker}(s t) \subseteq \mathcal{B}_{2}(\mathbb{C}), \sum m_{j} \rho\left(a_{j}\right) \in Z^{2}$ (pt., 3$)$ may be completed to a higher Chow cycle $\mathcal{Z}$ by adding 'decomposable' elements $\in Z^{1}$ (pt., 2$) \wedge Z^{1}$ (pt., 1). The composition $\pi_{\mathbb{R}} \circ \Phi_{2,3}$ on $\mathcal{Z}$ is then computed by $\Im\left(R_{\mathcal{Z}}\right)=\sum m_{j} D_{2}\left(a_{j}\right) \in \mathbb{R}$.

So if $\sum m_{j} D_{2}\left(a_{j}\right) \neq 0$ (there are many examples), $A J(\mathcal{Z}) \in \mathbb{C} / \mathbb{Z}(2)$ is nontorsion.

Example. Let $X$ be a compact Riemann surface. We recover the formula for the real regulator

$$
r_{2,2}: C H^{2}(X, 2) \rightarrow H_{\mathcal{D}}^{2}(X, \mathbb{R}(2))
$$

in [Ram89] by composing $A J$ with $\pi_{\mathbb{R}}$ (which takes the imaginary part in this case).

The irreducible components of $\mathcal{Z} \in Z^{2}(X, 2)$ are of two types: (a) curves contained in $\square^{2}$ over isolated points of $X$; (b) graphs (over $X$ ) of pairs of meromorphic functions $f, g \in \mathbb{C}(X)$. Writing

$$
\Gamma_{f, g}:=\left\{(x, f(x), g(x)) \in X \times\left(\mathbb{P}^{1}\right)^{2} \mid x \in X\right\} \cap\left(X \times \square^{2}\right),
$$

one has

$$
\mathcal{Z}=\sum m_{k} \cdot\left\{x_{k}\right\} \times C_{k}+\sum n_{j} \Gamma_{f_{j}, g_{j}}=\mathcal{Z}_{(a)}+\mathcal{Z}_{(b)}
$$




\section{KerR, J. D. LeWis And S. MÜller-Stach}

Now assume $\partial_{\mathcal{B}} \mathcal{Z}=0$, and compute $A J[\mathcal{Z}]$ : since $\int_{C_{j}} R^{2} \wedge \pi_{X}^{*} \omega=0$ (for any 1-form on $X$ ), $R_{\mathcal{Z}_{(a)}}=0$. Thus

$$
R_{\mathcal{Z}}=R_{\mathcal{Z}_{(b)}}=\sum n_{j} R_{f_{j}, g_{j}}=\sum n_{j}\left(\log f_{j} \operatorname{dlog} g_{j}-2 \pi \mathrm{i} \log g_{j} \delta_{T_{f_{j}}}\right) ;
$$

integrating the latter current over loops $\gamma \in \Omega X$ gives

$$
A J[\mathcal{Z}] \in \operatorname{Hom}\left(H_{1}(X, \mathbb{Z}), \mathbb{C} / \mathbb{Z}(2)\right) \cong H_{\mathcal{D}}^{1}(X, \mathbb{Z}(2)) .
$$

The technical point here is this: prior to integrating one should move the loop (in its homology class) to avoid all points $\left\{x_{k}\right\}$ and $\bigcup\left|\left(f_{j}\right)\right| \cup\left|\left(g_{j}\right)\right|$. It can be shown directly (and is also clear from the setup here) that integrals over loops around any of these points are trivial $(\in \mathbb{Z}(2))$, and so the integral is independent of the 'move' in question. Note that the imaginary part of $\log f \operatorname{dlog} g-2 \pi \mathrm{i} \log g \delta_{T_{f}}$ is $\log |f| \operatorname{darg} g+\arg f \operatorname{dlog}|g|-2 \pi \log |g| \delta_{T_{f}}$. Adding to this d $[-\arg f \log |g|]=-\log |g| \operatorname{darg} f-$ $\arg f \operatorname{dlog}|g|+2 \pi \log |g| \delta_{T_{f}}$ gives the cohomologous current

$$
\log |f| \operatorname{darg} g-\log |g| \operatorname{darg} f .
$$

(It is well known that Beilinson refers to this as 'Mama's formula'.) Integrating this over loops gives

$$
r_{2,2}[\mathcal{Z}] \in \operatorname{Hom}\left(H_{1}(X, \mathbb{Z}), \mathbb{R}\right) \cong H_{\mathcal{D}}^{2}(X, \mathbb{R}(2)) .
$$

An alternative formula sends

$$
\gamma \mapsto \Im\left(\int_{\gamma} \log f \frac{d g}{g}-\log |g(p)| \int_{\gamma} \frac{d f}{f}\right),
$$

where $\gamma$ is based at $p$ and $\log f$ is not the principal branch (but rather is continued along $\gamma$, starting from $p$ ).

\subsection{Geometric interpretation}

Now we ask, to what extent is $A J$ (for $n \geqslant 1$ ) 'like' an Abel-Jacobi map in the classical sense (for $n=0$ ), of integrating forms over a chain $\Gamma$ with $\partial \Gamma=\mathcal{Z}$ ? To answer this question we first extend the classical approach to the subgroup $Z^{p}\left(X \times \square^{n}, X \times \partial \square^{n}\right) \subseteq c^{p}(X, n)$ of relative (algebraic) cycles, consisting of those $\mathcal{Z}$ for which $\mathcal{Z} \cdot\left(X \times \partial \square^{n}\right)=0$, i.e. the intersections with each face cancel (counted with multiplicity). Since every class in $C H^{p}(X, n)$ is represented by such a cycle, it makes sense to ask whether the resulting relative $A J$ map coincides with the $A J$ constructed above.

The relevant details in the discussion that follows can be found in [Ker03]. Let $\mathcal{Z} \in Z^{p}(X \times$ $\left.\square^{n}, X \times \partial \square^{n}\right)$ have [complex] dimension $d=m+n-p$. Assume as above that $[\mathcal{Z}] \in C H_{\text {hom }}^{p}(X, n)$, and for the time being that $\mathcal{Z}$ is also in $Z_{\mathbb{R}}^{p}(X, n)$. We replace $\mathcal{Z}$ by a 'limit' of topological cycles via a kind of excision. Namely, writing $\mathbb{I}^{n}:=\bigcup_{i=1}^{n}\left\{\left(z_{1}, \ldots, z_{n}\right) \in\left(\mathbb{C}^{*}\right)^{n} \mid z_{i}=1\right\}$ and letting $\mathcal{Z}_{\epsilon}^{0}$ represent the analytic closure of $\mathcal{Z} \cap\left(X \times \square_{\epsilon}^{n}\right)$ on $X \times\left(\mathbb{C}^{*}\right)^{n}$, for each $\epsilon>0$ (sufficiently small) there are topological relative cycles

$$
\mathcal{Z}_{\epsilon}^{0}+\mathcal{W}_{\epsilon}=\mathcal{Z}_{\epsilon} \in Z_{2 d}^{\text {top }}\left(X \times\left(\mathbb{C}^{*}\right)^{n}, X \times \mathbb{I}^{n}\right)
$$

where

$$
\lim _{\epsilon \rightarrow 0} \int_{\mathcal{W}_{\epsilon}} \pi_{\square}{ }^{*}\left\{\begin{array}{c}
\Omega^{n} \\
R^{n}
\end{array}\right\} \wedge \pi_{X}{ }^{*} \alpha=0 \quad\left(\forall C^{\infty} \text {-forms } \alpha \text { on } X\right) .
$$

Since Lefschetz duality guarantees a perfect pairing between

$$
H_{2 d}\left(X \times\left(\mathbb{C}^{*}\right)^{n}, X \times \mathbb{I}^{n}\right) \cong H_{2 d-n}(X) \otimes\left\langle\left(S^{1}\right)^{n}\right\rangle
$$

and

$$
H_{2 p}\left(X \times \square^{n}, X \times \partial \square^{n}\right) \cong H_{2 p-n}(X) \otimes\left\langle T^{n}\right\rangle
$$


$\mathcal{Z}_{\epsilon}$ is homologous $\left(\bmod X \times \mathbb{I}^{n}\right)$ to $T_{\mathcal{Z}_{\epsilon}} \times\left(S^{1}\right)^{n}$, where $T_{\mathcal{Z}_{\epsilon}}=\pi_{X}\left(\mathcal{Z}_{\epsilon} \cap\left(X \times T^{n}\right)\right)$. So there exists a topological $(2 d+1)$-chain $\Gamma_{\epsilon}^{0}$ on $X \times\left(\mathbb{C}^{*}\right)^{n}$ such that

$$
\partial \Gamma_{\epsilon}^{0}-\mathcal{Z}_{\epsilon}+T_{\mathcal{Z}_{\epsilon}} \times\left(S^{1}\right)^{n} \subseteq X \times \mathbb{I}^{n} .
$$

The relative cycle $\mathcal{Z}$ is also a higher Chow cycle; therefore $T_{\mathcal{Z}_{\epsilon}}\left[\sim T_{\mathcal{Z}}\right] \sim 0$ is the boundary of a membrane $\zeta_{\epsilon}$ on $X$. (This is equivalent to $[\mathcal{Z}] \in C H_{\text {hom }}^{p}(X, n)$, which is what we are assuming.)

Set $\Gamma_{\epsilon}=\Gamma_{\epsilon}^{0}+\zeta_{\epsilon} \times\left(S^{1}\right)^{n}$. Since this has boundary $\mathcal{Z}_{\epsilon}\left(\bmod X \times \mathbb{I}^{n}\right)$, it now makes sense to define the relative Abel-Jacobi of $\mathcal{Z}$ as a functional (mod periods) on

$$
F^{d+1} H^{2 d+1}\left(X \times\left(\mathbb{C}^{*}\right)^{n}, X \times \mathbb{I}^{n} ; \mathbb{C}\right) \cong F^{d-n+1} H^{2 d-n+1}(X, \mathbb{C}) \otimes\left\langle\frac{1}{(2 \pi \mathrm{i})^{n-p}} \Omega^{n}\right\rangle
$$

induced by the formula

$$
A J_{\text {rel }}(\mathcal{Z}) \omega:=\lim _{\epsilon \rightarrow 0} \frac{1}{(2 \pi \mathrm{i})^{n-p}} \int_{\Gamma_{\epsilon}} \pi_{\square} \Omega^{n} \wedge \pi_{X}{ }^{*} \omega
$$

where as test forms we use all d-closed $\omega \in F^{m-p+1} \Omega_{\mathcal{X}^{\infty}}^{2 m-2 p+n+1}(X)$. It can be shown that the resulting map

$$
\begin{aligned}
A J_{\mathrm{rel}} & : Z^{p}\left(X \times \square^{n}, X \times \partial \square^{n}\right) \rightarrow H_{\mathcal{D}}^{2 p-n}(X, \mathbb{Z}(p)) \\
\cong & \frac{H^{2 p-n-1}(X, \mathbb{C})}{F^{p} H^{2 p-n-1}(X, \mathbb{C})+H^{2 p-n-1}(X, \mathbb{Z}(p))}
\end{aligned}
$$

respects relative rational equivalence. (Intuitively speaking, $A J_{\text {rel }}$ should go to some ' $H_{\mathcal{D}}^{2 p}(X \times$ $\left.\square^{n}, X \times \partial \square^{n} ; \mathbb{Z}(p)\right)^{\prime}$; one can easily justify defining this to be $H_{\mathcal{D}}^{2 p-n}(X, \mathbb{Z}(p))$.)

To see that this is the same as $A J$ of $\mathcal{Z}$ (considered instead as a higher Chow cycle), we show the following proposition.

Proposition 5.1. We have

$$
\lim _{\epsilon \rightarrow 0} \int_{\Gamma_{\epsilon}} \Omega^{n} \wedge \omega=(-1)^{n}\left(\int_{\mathcal{Z}} R^{n} \wedge \omega+(-2 \pi \mathrm{i})^{n} \int_{\zeta} \omega\right)
$$

for $\omega$ d-closed.

Proof. By Stokes' theorem for currents and (5.2),

$$
\begin{aligned}
\int_{\mathcal{Z}_{\epsilon}\left(=\partial \Gamma_{\epsilon}\right)} R^{n} \wedge \omega & =(-1)^{n} \int_{\Gamma_{\epsilon}} \mathrm{d}\left[R^{n} \wedge \omega\right] \\
& =(-1)^{n} \int_{\Gamma_{\epsilon}} \mathrm{d}\left[R^{n}\right] \wedge \omega \\
& =(-1)^{n} \int_{\Gamma_{\epsilon}} \Omega^{n} \wedge \omega-(-2 \pi \mathrm{i})^{n} \int_{\pi_{X}\left[\Gamma_{\epsilon} \cap\left(X \times T^{n}\right)\right]} \omega-0 .
\end{aligned}
$$

The residue term in (5.2) makes no contribution because $\Gamma_{\epsilon} \cap\left(X \times \partial \square^{n}\right)=\emptyset$. Now one can construct $\Gamma_{\epsilon}^{0}$ explicitly (as in [Ker03]) so that

$$
\operatorname{dim}_{\mathbb{R}}\left[\pi_{X}\left\{\Gamma_{\epsilon}^{0} \cap\left(X \times T^{n}\right)\right\}\right]<2 d-n+1 ;
$$

for our purposes then $\pi_{X}\left[\Gamma_{\epsilon} \cap\left(X \times T^{n}\right)\right]=\zeta_{\epsilon}$, and

$$
\int_{\Gamma_{\epsilon}} \omega \wedge \Omega^{n}=(-1)^{n}\left(\int_{\mathcal{Z}_{\epsilon}} \omega \wedge R^{n}+(-2 \pi \mathrm{i})^{n} \int_{\zeta_{\epsilon}} \omega\right) .
$$

Taking limits then gives the result. 


\section{KerR, J. D. Lewis AND S. MÜLler-StaCH}

Remark. (i) In light of the coincidence of these two maps, we may view the previous 'simplification for $n \geqslant p$ or $p>m$ ' as asserting that the relative $A J$ map on $\left(X \times \square^{n}, X \times \partial \square^{n}\right)$ may be 'pushed down to $X^{\prime}$ where it consists merely of computing periods of the current $R_{\mathcal{Z}}$. This is essentially thanks to the fact that $H^{n}\left(\left(\mathbb{C}^{*}\right)^{n}, \mathbb{I}^{n}\right)=F^{n} H^{n}\left(\left(\mathbb{C}^{*}\right)^{n}, \mathbb{I}^{n}\right)$, and $H^{*}\left(\left(\mathbb{C}^{*}\right)^{n}, \mathbb{I}^{n}\right)=0$ for $* \neq n$.

(ii) We can modify this approach in case $\mathcal{Z} \notin Z_{\mathbb{R}}^{p}(X, n)$. Referring to the Proposition in $\S 5.4$, we remark that $\mathcal{Z}$ can also be brought into good position with respect to the $X \times\left(T_{z_{1}} \cap \cdots \cap T_{z_{i}}\right)$ etc. by perturbing these real chains (rather than $\mathcal{Z}$ ) by $\tau$.

To deal with such $\mathcal{Z}$, therefore, one merely repeats all of $\S 5.8$ with the 'perturbation' $T_{z_{i}} \mapsto$ $T_{z_{i}}^{\prime}:=T_{z_{i} / \alpha_{i}}$ (which accordingly leads to a different chain $\Gamma_{\epsilon}^{\prime}$ ), and also with branches of $\log \left(z_{i}\right)$ in $R^{n}$ replaced by branches with cuts at $T_{z_{i}}^{\prime}$. Proposition 5.1 then holds exactly. There is no need to take limits as the perturbations approach 1 , since $\Gamma_{\epsilon}^{\prime}$ amounts simply to a different choice of bounding membrane for computing the relative $A J$ map.

Such a procedure has been carried out in a concrete computation in [Ker03, § 3.2].

\subsection{Quasiprojective case}

Now let $V \subset X$ be an arbitrary divisor; we show how to define

$$
A J_{X \backslash V}: C H^{p}(X \backslash V, n) \rightarrow H_{\mathcal{D}}^{2 p-n}(X \backslash V, \mathbb{Z}(p)) .
$$

Let $(\bar{X}, \bar{V})$ be a pair where $\bar{V}$ is a normal crossing (n.c.) divisor and $\bar{X} \backslash \bar{V}=X \backslash V$. By Bloch's moving lemma, restriction induces a quasiisomorphism of complexes

$$
Z^{p}(\bar{X},-\bullet) / Z^{p-1}(\bar{V},-\bullet) \underset{\simeq}{\longrightarrow} Z^{p}(X \backslash V,-\bullet) .
$$

The homotopy $\mathcal{H}$ described in $\S 5.4$ leads to a proof that

$$
Z_{\mathbb{R}}^{p}(\bar{X},-\bullet) / Z_{\mathbb{R}}^{p-1}(\bar{V},-\bullet) \stackrel{\simeq}{\longrightarrow} Z^{p}(\bar{X},-\bullet) / Z^{p-1}(\bar{V},-\bullet) .
$$

Finally, the triple $\left((2 \pi \mathrm{i})^{n} T_{\mathcal{Z}}, \Omega_{\mathcal{Z}}, R_{\mathcal{Z}}\right)$ once again yields a map of complexes

$$
\begin{aligned}
A J_{X \backslash V}: Z_{\mathbb{R}}^{p}(\bar{X},-\bullet) / Z_{\mathbb{R}}^{p-1}(\bar{V},-\bullet) \\
\quad \longrightarrow \text { Cone }\left\{\begin{array}{c}
\mathcal{C}_{2 m-2 p-\bullet}(\bar{X}, \bar{V} ; \mathbb{Z}(p)) \\
\oplus \\
\Gamma\left(F^{p \prime} \mathcal{D}_{\bar{X}}^{2 p+\bullet}(\log \bar{V})\right)
\end{array} \rightarrow \Gamma\left({ }^{\prime} \mathcal{D}_{\bar{X}}^{2 p+\bullet}(\log \bar{V})\right)\right\}[-1](-d),
\end{aligned}
$$

which induces $A J_{X \backslash V}$.

Remark. (i) We need the n.c. condition in order that the latter complex actually compute $H_{\mathcal{D}}^{2 p-n}(X \backslash$ $V, \mathbb{Z}(p))$.

(ii) We could not use $Z^{p}(\bar{X} \backslash \bar{V},-\bullet)$ here because $\Omega_{\mathcal{Z}}$ and $R_{\mathcal{Z}}$ have (in general) worse than log poles along $\bar{V}$, for $\mathcal{Z} \in Z^{p}(\bar{X} \backslash \bar{V}, n)$.

(iii) The simplifications that occurred in the projective case for $n \geqslant 1$ (and rational coefficients) require $p>m$ or $n>p$ here $\left(\operatorname{as} F^{p} H^{2 p-n}(X \backslash V, \mathbb{C}) \cap H^{2 p-n}(X \backslash V, \mathbb{Q}(p))\right.$ must vanish).

(iv) In case $n>p$, or $n=p>m$, the $\Gamma\left(F^{p \prime} \mathcal{D}_{\bar{X}}^{2 p+\bullet}(\log \bar{V})\right)$ terms do not enter. The situation simplifies and we may work with $R_{\mathcal{Z}}$ (respectively $T_{\mathcal{Z}}$ ) directly on $X \backslash V$ (respectively $(X, V)$ ). Taking the limit we get $A J$ maps over the generic point

$$
A J_{\eta_{X}}: C H^{p}(\mathbb{C}(X), n) \rightarrow H_{\mathcal{D}}^{2 p-n}\left(\eta_{X}, \mathbb{Z}(p)\right) .
$$

Example. For $n=p>m, C H^{n}(\mathbb{C}(X), n) \cong K_{n}^{M}(\mathbb{C}(X))$ by a result of Totaro [Tot92] (this is also due to Nesterenko and Suslin). The resulting map

$$
A J_{\eta_{X}}: K_{n}^{M}(\mathbb{C}(X)) \rightarrow H_{\mathcal{D}}^{n}\left(\eta_{X}, \mathbb{Q}(n)\right) \cong H^{n-1}\left(\eta_{X}, \mathbb{C} / \mathbb{Q}(n)\right)
$$


is called the Milnor regulator and is studied extensively in [Ker03]. As in the projective case, $A J_{\eta_{X}}\left\{f_{1}, \ldots, f_{n}\right\}$ may be computed as a functional on topological cycles, namely

$$
\int_{(\cdot)} R\left(f_{1}, \ldots, f_{n}\right) \in \operatorname{Hom}\left(H_{n-1}\left(\eta_{X}, \mathbb{Z}\right), \mathbb{C} / \mathbb{Q}(n)\right) .
$$

\section{How a cycle gives rise to an extension of motives (third construction of $A J$ )}

Let $\mathcal{Z}$ be a cycle in $C H_{\text {hom }}^{p}(X, n)$. By normalization of chain complexes [EZ50, p. 512], we may assume that all individual intersections $\mathcal{Z} \cap\left\{z_{i}=0, \infty\right\}$ are zero. Let $U:=\square_{X}^{n} \backslash|\mathcal{Z}|$ and $\partial U:=$ $U \cap \partial \square_{X}^{n}$. One has an exact sequence (in any reasonable theory satisfying weak purity and the homotopy axiom)

$$
H^{2 p-2}(U) \rightarrow H^{2 p-2}(\partial U) \rightarrow H^{2 p-1}(U, \partial U) \rightarrow H^{2 p-1}(U) \rightarrow H^{2 p-1}(\partial U) .
$$

By weak purity $H_{|\mathcal{Z}|}^{i}\left(\square_{X}^{n}\right)=0$ for $i<2 p$. Also by the homotopy axiom, $H^{i}\left(\square_{X}^{n}\right)=H^{i}(X)$ for $i \geqslant 0$. Therefore $H^{2 p-2}(U)=H^{2 p-2}(X)$ (naturally) and furthermore one has for all $i \geqslant 0$

$$
H^{i}\left(\partial \square_{X}^{n}\right)=H^{i}(X) \oplus H^{i-n+1}(X),
$$

i.e. $\partial \square_{X}^{n}$ is like a real $(n-1)$-sphere. Moreover $H^{i}(\partial U)=H^{i}\left(\partial \square_{X}^{n}\right)$ for $i=2 p-2$, again by weak purity for the faces. Using all this, the long exact sequence now becomes

$$
0 \rightarrow H^{2 p-n-1}(X) \rightarrow H^{2 p-1}(U, \partial U) \rightarrow \operatorname{ker}\left\{H^{2 p-1}(U) \rightarrow H^{2 p-1}(\partial U)\right\} \rightarrow 0 .
$$

But

$$
\operatorname{ker}\left\{H^{2 p-1}(U) \rightarrow H^{2 p-1}(\partial U)\right\} \subseteq \operatorname{ker}\left\{H_{|\mathcal{Z}|}^{2 p}\left(\square_{X}^{n}\right)^{\circ} \stackrel{\beta}{\rightarrow} H_{|\partial \mathcal{Z}|}^{2 p}\left(\partial \square_{X}^{n}\right)^{\circ}\right\}
$$

The symbol ${ }^{\circ}$ stands for the kernel of the map forgetting supports. This implies that we have a long exact sequence

$$
0 \rightarrow H^{2 p-n-1}(X) \rightarrow H^{2 p-1}(U, \partial U) \rightarrow \operatorname{ker}(\beta) \rightarrow H^{2 p-n}(X) .
$$

To see this, one simply applies the serpent lemma to the following diagram of exact sequences.

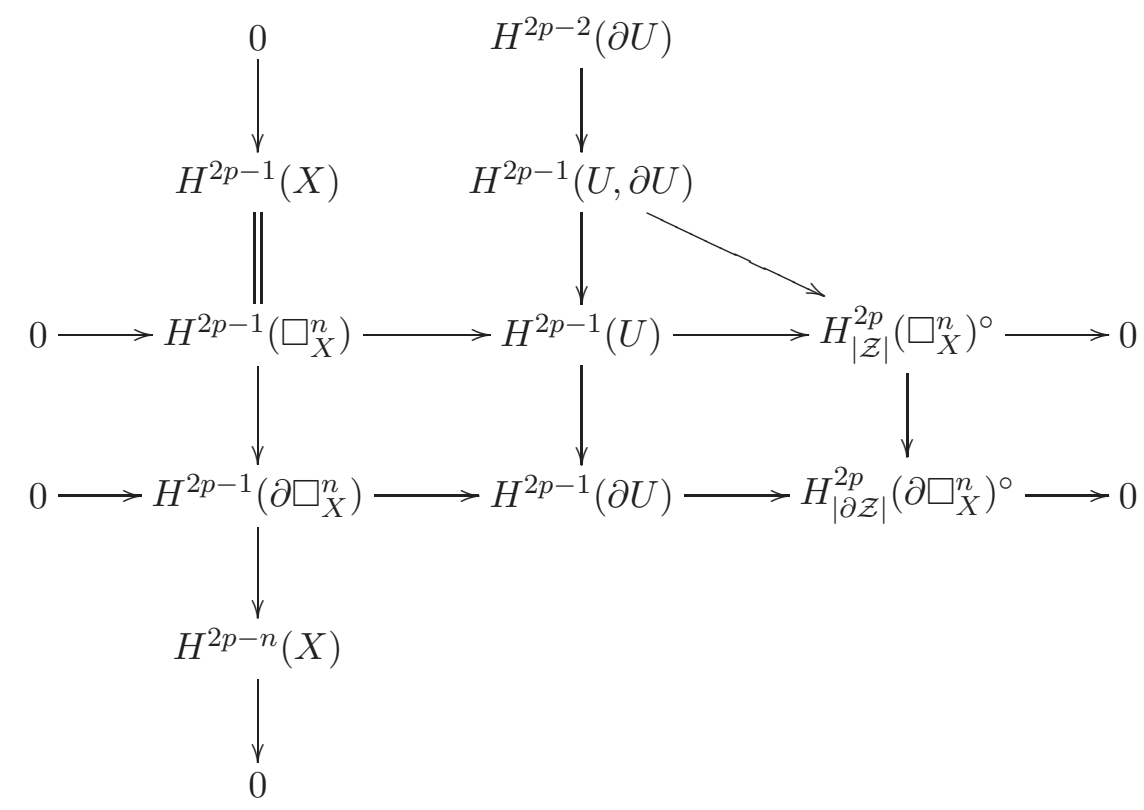

This yields an extension via pullback

$$
0 \rightarrow H^{2 p-n-1}(X, \mathbb{Z}(p)) \rightarrow \mathbf{E} \rightarrow \mathbb{Z}(0) \rightarrow 0,
$$




\section{KerR, J. D. Lewis AND S. MÜlleR-StaCH}

where the motive $\mathbb{Z}(0)$ is generated by the algebraic cycle $\{\mathcal{Z}\}$ in $\operatorname{ker}(\beta)$. If we specialize to singular cohomology, we obtain an extension of mixed Hodge structures:

$$
\mathbf{E} \in \operatorname{Ext}_{\mathrm{MHS}}^{1}\left(\mathbb{Z}(0), H^{2 p-n-1}(X, \mathbb{Z}(p))\right) \simeq \frac{H^{2 p-n-1}(X, \mathbb{C})}{F^{p} H^{2 p-n-1}(X, \mathbb{C})+H^{2 p-n-1}(X, \mathbb{Z}(p))}=: J^{p, n}(X),
$$

as desired.

Note that $H_{|\mathcal{Z}|}^{2 p}\left(\square_{X}^{n}\right)$ is generated by the components of $|\mathcal{Z}|$, and therefore $F^{p} H_{|\mathcal{Z}|}^{2 p}\left(\square_{X}^{n}\right)=$ $H_{|\mathcal{Z}|}^{2 p}\left(\square_{X}^{n}\right)$. Now put

$$
V=\operatorname{ker}\left\{\operatorname{ker}(\beta) \rightarrow H^{2 p-n}(X)\right\} .
$$

Then $F^{p} V=V$. By applying the serpent lemma to

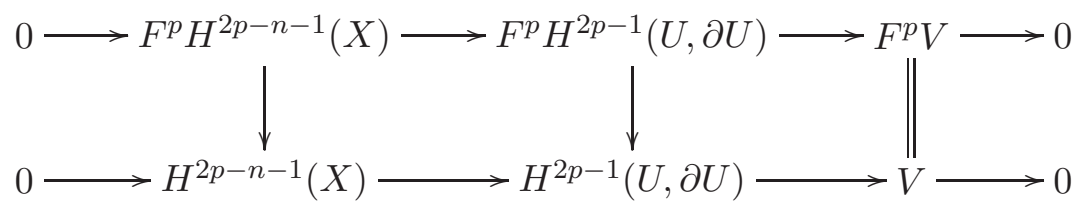

we deduce that

$$
\frac{H^{2 p-n-1}(X, \mathbb{C})}{F^{p} H^{2 p-n-1}(X, \mathbb{C})} \simeq \frac{H^{2 p-1}(U, \partial U, \mathbb{C})}{F^{p} H^{2 p-1}(U, \partial U, \mathbb{C})},
$$

and hence

$$
J^{p, n}(X) \simeq \frac{H^{2 p-1}(U, \partial U, \mathbb{C})}{F^{p} H^{2 p-1}(U, \partial U, \mathbb{C})+H^{2 p-n-1}(X, \mathbb{Z}(p))} .
$$

Note that the formula in (6.4) generalizes the formula in (3.3) for the case $n=0\left(\square_{X}^{0}=X\right.$, $\left.\partial \square_{X}^{0}=0\right)$. From (6.1), we have a sequence analogous to (3.1), namely

$$
0 \rightarrow H^{2 p-n-1}(X, \mathbb{Z}(p)) \stackrel{\tilde{\beta}}{\rightarrow} H^{2 p-1}(U \backslash \partial U, \mathbb{Z}(p)) \rightarrow \operatorname{ker}(\beta) \rightarrow H^{2 p-n}(X, \mathbb{Z}(p)),
$$

and modulo the image of $\tilde{\beta}$, the higher Chow cycle $\mathcal{Z} \in C H_{\text {hom }}^{p}(X, n)$ defines a class $\Psi_{p, n}(\mathcal{Z}) \in$ $J^{p, n}(X)$, with the help of (6.4).

\section{Comparing definitions}

In this section it is proved that the constructions of $A J$ via explicit currents and via extension classes (in $\S \S 5$ and 6 , respectively) agree, namely the following theorem holds.

Theorem 7.1. For $X$ projective and $[\mathcal{Z}] \in C H_{\mathrm{hom}}^{p}(X, n), \Psi_{p, n}(\mathcal{Z})=\Phi_{p, n}(\mathcal{Z})$. If $n \geqslant 1$, then without the 'hom' assumption one has this equality modulo torsion (i.e. replacing $\mathbb{Z}$ by $\mathbb{Q}$ in the target groups).

Proof. We only need to show that the extension definition gives the same as our explicit formula, since the equality between Bloch's map and the extension definition was already shown by Scholl [Sch93]. We begin by picking apart the last section's construction in some detail, and show in particular that this construction agrees with the geometric interpretation of the $A J$ map given in $\S 5.8$ above.

Recall $\square^{n}=\left(\mathbb{P}^{1} \backslash\{1\}\right), \partial \square^{n}=\operatorname{Alt}_{n}\left(\{0, \infty\} \times\left(\mathbb{P}^{1} \backslash\{1\}\right)^{n-1}\right), \square_{X}^{n}=X \times \square^{n}$, etc. Let $[\mathcal{Z}]$ be any class $\in C H^{p}(X, n)$. By normalization [EZ50] we may choose $\mathcal{Z}$ to be a relative cycle, so that all face intersections $\mathcal{Z} \cdot \partial \square_{X}^{n}$ are zero as cycles; moreover, $H^{2 p}\left(\square_{X}^{n}\right)$ injects into $H^{2 p}\left(\partial \square_{X}^{n}\right)$ (e.g. see diagram (6.2)). So the fundamental class of $\mathcal{Z}$ in $H_{|\mathcal{Z}|}^{2 p}\left(\square_{X}^{n}\right)$ goes to zero in $H^{2 p}\left(\square_{X}^{n}\right)$ and $H_{|\partial \mathcal{Z}|}^{2 p}\left(\partial \square_{X}^{n}\right)$; this 
accounts for $\mathcal{Z}$ determining a class in $\operatorname{ker}(\beta)$, where

$$
\beta: H_{|\mathcal{Z}|}^{2 p}\left(\square_{X}^{n}\right)^{\circ} \rightarrow H_{|\partial \mathcal{Z}|}^{2 p}\left(\partial \square_{X}^{n}\right)^{\circ} .
$$

None of this has anything to do with $[\mathcal{Z}] \in C H_{\text {hom }}^{p}(X, n)$.

Now we describe the map

$$
\operatorname{ker}(\beta) \rightarrow H^{2 p-n}(X) ;
$$

triviality of this map is what the 'hom' indicates. Roughly speaking, this can be seen as also saying that the fundamental class of $\mathcal{Z}$ in $H^{2 p}\left(\square_{X}^{n}, \partial \square_{X}^{n}\right)$ is trivial. This corresponds to triviality of $\mathcal{Z}$ as a topological cycle in $H_{2 m+2 n-2 p}\left(X \times\left(\mathbb{C}^{*},\{1\}\right)^{n}\right)$, which is best expressed by casting $\mathcal{Z}$ as a limit of topological cycles $\mathcal{Z}_{\epsilon}$ which are (modulo $\left.X \times \mathbb{I}^{n}\right)$ boundaries of topological $(2 m+2 n-2 p+1$ )-chains compactly supported on $X \times\left(\mathbb{C}^{*}\right)^{n}$.

This map was defined via the following 'serpent' of maps and lifts.

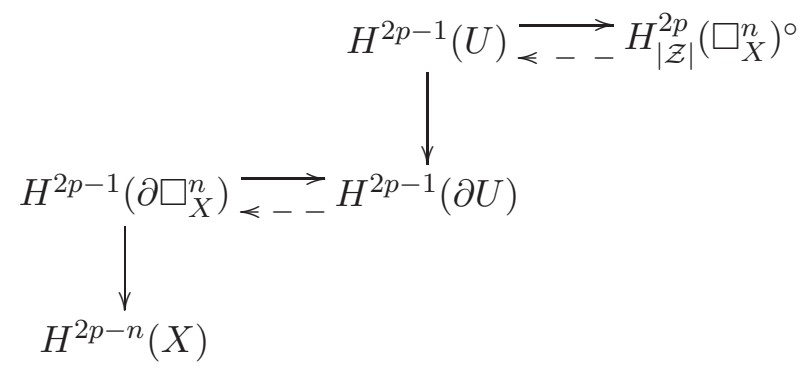

Here the last (vertical) map takes

$$
H^{2 p-1}\left(\partial \square_{X}^{n}\right) \cong H^{0}\left(\partial \square^{n}\right) \otimes H^{2 p-1}(X) \oplus H^{n-1}\left(\partial \square^{n}\right) \otimes H^{2 p-n}(X) \rightarrow H^{2 p-n}(X),
$$

which is to say $\alpha \mapsto \pi_{*}\left(\alpha \wedge \mathrm{d}\left[\Omega^{n}\right]\right)$ or (for topological cycles) $\mathcal{C} \rightarrow \pi_{*}\left(\mathcal{C} \cdot \partial T_{X}^{n}\right)$. [The point is that $H_{\left|\partial \square^{n \mid}\right|}^{n-1}\left(\left(\mathbb{P}^{1}\right)^{n}\right) \cong\left\{H^{n-1}\left(\partial \square^{n}\right)\right\}^{\vee}$ is generated by $\partial\left[\Omega^{n}\right]$ (or Poincaré-dually by $\left.\partial T^{n}\right)$; therefore wedging with this and pushing down (integrating fiberwise) 'removes' the $H^{n-1}\left(\partial \square^{n}\right)$ part from $\alpha$.] To see where $\mathcal{Z}$ goes under the 'composition', we will use the following equivalent homological serpent.

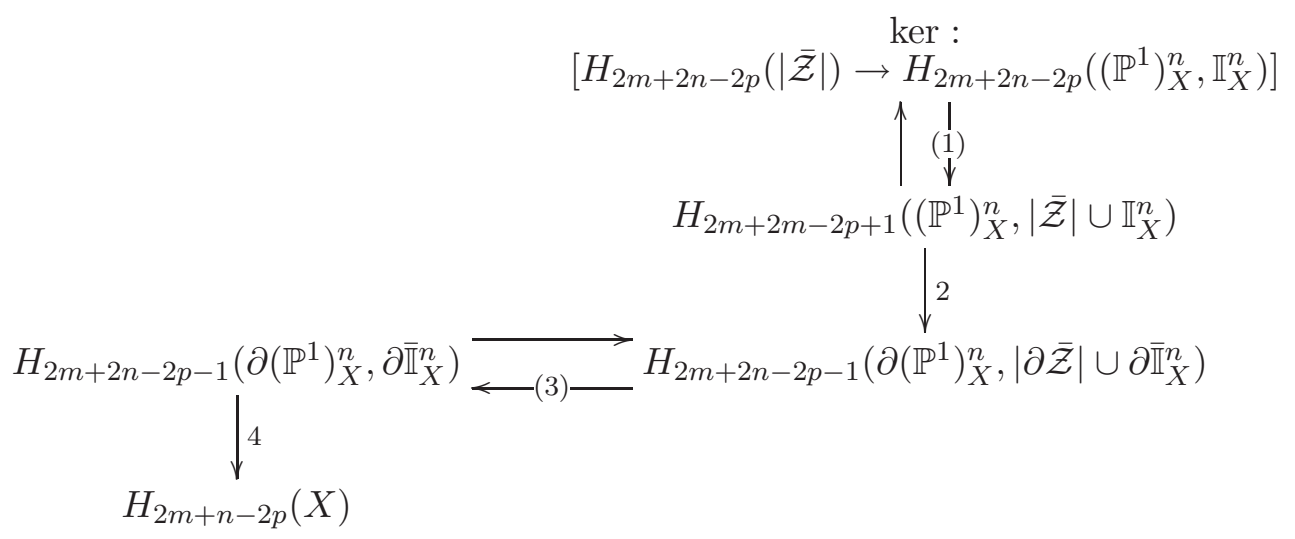

Tracing through, we have (since $\mathcal{Z}$ is in the upper right-hand kernel)

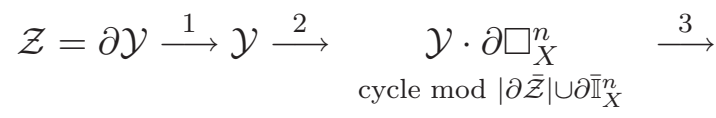

$$
\begin{aligned}
& \underset{\mathcal{Y} \cdot \partial \square_{X}^{n}}{\mathcal{Y}} \stackrel{4}{\longrightarrow} \pi_{*}\left(\left(\mathcal{Y} \cdot \partial \square_{X}^{n}\right) \cdot \partial T_{X}^{n}\right)=\pi_{*}\left(\mathcal{Y} \cdot \partial T_{X}^{n}\right) .
\end{aligned}
$$




\section{KerR, J. D. Lewis AND S. MÜlleR-StaCH}

Now recall that $T_{\mathcal{Z}}=\pi_{*}\left(\mathcal{Z} \cdot T_{X}^{n}\right)\left(\right.$ where $\left.T_{X}^{n}=X \times T^{n}\right)$. Since

$$
\partial \pi_{*}\left(\mathcal{Y} \cdot T_{X}^{n}\right)=\pi_{*}\left(\partial \mathcal{Y} \cdot T_{X}^{n}\right) \pm \pi_{*}\left(\mathcal{Y} \cdot \partial T_{X}^{n}\right),
$$

we see that the image of $\mathcal{Z}$ under the above map is (mod coboundary and up to sign) equivalent to $T_{\mathcal{Z}}$. Therefore we will write

$$
T: \operatorname{ker}(\beta) \rightarrow H^{2 p-n}(X)
$$

(or, more formally, $(2 \pi \mathrm{i})^{p} T: \operatorname{ker}(\beta) \rightarrow H^{2 p-n}(X, \mathbb{Z}(p))$ ).

If $[\mathcal{Z}] \in C H_{\text {hom }}^{p}(X, n)$ then $T_{\mathcal{Z}} \sim 0$. Thus we get an element in the right-hand term of the twisted sequence (from $(6.5)$

$$
0 \rightarrow H^{2 p-n-1}(X, \mathbb{Z}(p)) \stackrel{A}{\longrightarrow} H^{2 p-1}(U, \partial U)_{\mathbb{Z}(p)} \stackrel{B}{\longrightarrow} \operatorname{ker}(T)_{\mathbb{Z}(p)} \rightarrow 0 ;
$$

we show how to lift it to the center term. The map $B$ is the composite

$$
H^{2 p-1}(U, \partial U) \rightarrow H^{2 p-1}(U) \rightarrow H_{|\mathcal{Z}|}^{2 p}\left(\square_{X}^{n}\right)
$$

or (homologically)

$$
\begin{aligned}
& H_{2 m+2 n-2 p}(|\overline{\mathcal{Z}}|) \\
& \begin{array}{lc}
\uparrow & \mid \\
\partial & (1) \\
\mid & \downarrow
\end{array} \\
& H_{2 m+2 n-2 p+1}\left(X \times\left(\mathbb{C}^{*}\right)^{n},|\mathcal{Z}| \cup \mathbb{I}_{X}^{n}\right) \longrightarrow H_{2 m+2 n-2 p+1}\left(X \times\left(\mathbb{P}^{1}\right)^{n},|\overline{\mathcal{Z}}| \cup \overline{\mathbb{I}}_{X}^{n}\right)
\end{aligned}
$$

where the lifts indicated (possible because of the exact sequence) take $\mathcal{Z}$ to a bounding chain $\Gamma$ (where $\partial \Gamma=\mathcal{Z} \bmod \mathbb{I}_{X}^{n}$ ) and then to a limit of bounding chains $\Gamma_{\epsilon}$ compactly supported on $X \times\left(\mathbb{C}^{*}\right)^{n}\left(\right.$ with $\left.\partial \Gamma_{\epsilon}=\mathcal{Z}_{\epsilon} \bmod \mathbb{I}_{X}^{n}\right)$ as described in $\S 5$.

We will write $\zeta_{\mathcal{Z}}$ for the image of the integral lift

$$
\lim _{\epsilon \rightarrow 0} \Gamma_{\epsilon} \text { in } H^{2 p-1}(U, \partial U)_{\mathbb{C}} / F^{p} H^{2 p-1}(U, \partial U) .
$$

Its preimage $\tilde{\beta}^{-1}\left(\zeta_{\mathcal{Z}}\right)$ under

$$
\tilde{\beta}: \frac{H^{2 p-n-1}(X)_{\mathbb{C}}}{F^{p} H^{2 p-n-1}(X)} \cong \frac{H^{2 p-1}(U, \partial U)}{F^{p} H^{2 p-1}(U, \partial U)},
$$

taken modulo $H^{2 p-n-1}(X, \mathbb{Z}(p))$, gives $\Psi_{p, n}(\mathcal{Z})$. Now $\tilde{\beta}$ dualizes to

$$
\tilde{\beta}^{\vee}: F^{n+m-p+1} H^{2 n+2 m-2 p+1}\left(X \times\left(\mathbb{C}^{*}\right)^{n},|W| \cup \mathbb{I}_{X}^{n}\right)_{\mathbb{C}} \stackrel{(\cong)}{\longrightarrow} F^{m-p+1} H^{n+2 m-2 p+1}(X)_{\mathbb{C}} .
$$

If we think of $\tilde{\beta}^{-1}\left(\zeta_{\mathcal{Z}}\right) \in\left\{F^{m-p+1} H^{2 m-2 p+n+1}(X)_{\mathbb{C}}\right\}^{\vee}$ as a functional on forms, then it (and hence $\left.\Psi_{p, n}(\mathcal{Z})\right)$ is computed on $\omega$ by

$$
\left[\tilde{\beta}^{-1}\left(\zeta_{\mathcal{Z}}\right)\right] \omega=\zeta_{\mathcal{Z}}\left[\left(\tilde{\beta}^{\vee}\right)^{-1} \omega\right] .
$$

(This essentially comes from Carlson's theory [Car87].)

It remains to trace through $\tilde{\beta}^{\vee}$. As $\tilde{\beta}$ is given by the composition

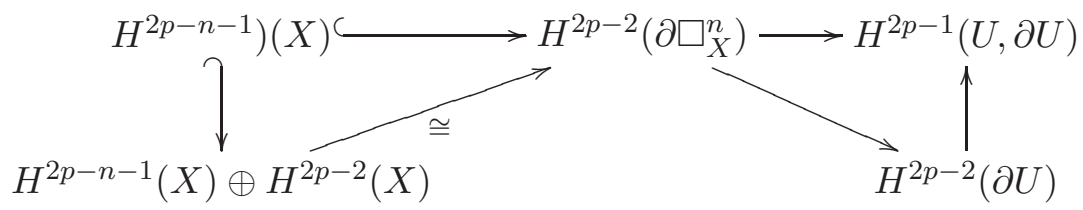

then $\tilde{\beta}^{\vee}$ must be

$$
H^{2 m-2 p+n+1}(X) \nVdash H_{\left|\partial \square_{X}^{n}\right|}\left(X \times\left(\mathbb{P}^{1}\right)^{n}\right) \stackrel{\mathrm{d}[\cdot]}{\longleftarrow} H^{2 m+2 n-2 p+1}\left(X \times\left(\mathbb{C}^{*}\right)^{n},|\mathcal{Z}| \cup \mathbb{I}_{X}^{n}\right),
$$




\section{The ABEL-JACOBI MAP FOR Higher ChOW GROUPS}

where the second map sends $\pi^{*} \omega \wedge \mathrm{d}\left[\Omega^{n}\right] \mapsto \omega$, and the group on the right-hand side we take to be represented by forms pulling back to 0 along $|\mathcal{Z}|$. If $\omega \in \Gamma\left(F^{m-p+1} \Omega_{X^{\infty}}^{2 m-2 p+n+1}\right)$ is (d-)closed then $\pi^{*} \omega \wedge \Omega^{n}$ is of type $F^{m+n-p+1}$ (whereas $\operatorname{dim}_{\mathbb{C}} \mathcal{Z}=m+n-p$ ), and so gives a lift of $\omega$ to the right-hand side; therefore we write $\left(\tilde{\beta}^{\vee}\right)^{-1} \omega=\pi^{*} \omega \wedge \Omega^{n}$. So $(*)$ is just

$$
\lim _{\epsilon \rightarrow 0} \int_{\Gamma_{\epsilon}} \pi_{1}^{*} \omega \wedge \pi_{2}^{*} \Omega^{n}
$$

identifying $\Psi_{p, n}(\omega)$ with the $A J$ for relative cycles $\in Z^{p}\left(\square_{X}^{n}, \partial \square_{X}^{n}\right)$ as described in $\S 5$. We already know this equates with $\Phi_{p, n}(\mathcal{Z})$ (or $A J(\mathcal{Z})$ ) by Proposition 5.1. This completes the proof of Theorem 7.1.

Remark. A few words need to be said regarding the ' $\lim _{\epsilon \rightarrow 0}$ '. The 'relative quasiprojective variety'

$$
(* *) \quad\left(X \times\left(\mathbb{C}^{*}\right)^{n},|\mathcal{Z}| \cup \mathbb{I}_{X}^{n}\right)
$$

is Poincaré-dual to $(U, \partial U)$; here these two play roles analogous to those played, respectively, by $(X,|\mathcal{Z}|)$ and $(X \backslash|\mathcal{Z}|)$ in $\S 3$. We need to be able to pair forms on (**) [which pull back to 0 along $|\mathcal{Z}| \cup \mathbb{I}_{X}^{n}$ but have poles along $\left.\partial \square_{X}^{n}\right]$ with topological cycles there; this is the reason for using limits of chains to compute homology (the integrals only make sense as a limit). Furthermore this needs to be done in such a way that coboundaries and topological cycles (respectively cocycles and topological boundaries) pair to zero; referring to $\S 5$, the fact that

$$
\lim _{\epsilon \rightarrow 0} \int_{\mathcal{W}_{\epsilon}} \pi_{\square}^{*}\left\{\begin{array}{c}
\Omega^{n} \\
R^{n}
\end{array}\right\} \wedge \pi_{X}^{*} \alpha=0 \quad \text { for any } C^{\infty} \text {-form } \alpha
$$

ensures that this condition is met (as far as needed for the arguments here).

\section{REFERENCES}

Bei85 A. Beilinson, Higher regulators and values of L-functions, J. Soviet Math 30 (1985), 2036-2070.

Blo86a S. Bloch, Algebraic cycles and higher K-theory, Adv. Math. 61 (1986), 267-304.

Blo86b S. Bloch, Algebraic cycles and the Beilinson conjectures, Contemp. Math. 58 (1986), 65-79.

Blo91 S. Bloch, Algebraic cycles and the Lie algebra of mixed Tate motives, J. Amer. Math. Soc. 4 (1991), 771-791.

Car87 J. Carlson, The geometry of the extension class of a mixed Hodge structure, in Algebraic geometry, Bowdoin, 1985, Proc. Symp. Pure Mathematics, vol. 46, part 2 (American Mathematical Society, Providence, RI, 1987), 199-222.

DS91 C. Deninger and A. Scholl, The Beilinson conjectures, in L-functions and arithmetic, eds J. Coates and M. J. Taylor, London Mathematical Society Lecture Note Series, vol. 153 (Cambridge University Press, Cambridge, 1991), 173-209.

EZ50 S. Eilenberg and J. A. Zilber, Semi-simplicial complexes and singular homology, Ann. of Math. (2) 51 (1950), 499-513.

EV88 H. Esnault and E. Viehweg, Deligne-Beilinson cohomology, in Beilinson's conjectures on special values of L-functions (Academic Press, Boston, 1988), 43-92.

Gon95 A. Goncharov, Chow polylogarithms and regulators, Math. Res. Lett. 2 (1995), 95-112.

Jan88 U. Jannsen, Deligne homology, Hodge D-conjecture, and motives, in Beilinson's conjectures on special values of L-functions (Academic Press, Boston, MA, 1988), 305-372.

Ker03 M. Kerr, Geometric construction of Regulator currents with applications to algebraic cycles, Thesis, Princeton University (2003).

Kin83 J. King, Log complexes of currents and functorial properties of the Abel-Jacobi map, Duke Math. J. 50 (1983), 1-53.

Lev88 M. Levine, Localization on singular varieties, Invent. Math. 31 (1988), 423-464. 


\section{The ABEL-JACOBI MAP FOR Higher ChOW GROUPS}

Lev98 M. Levine, Mixed motives, AMS Surveys and Monographs, vol. 57 (American Mathematical Society, Providence, RI, 1998).

Lev03 M. Levine, Motivic cohomology, Notes on lectures delivered at the Morelia Algebraic Geometry Conference, 23-24 June 2003 (unpublished).

Ram89 D. Ramakrishnan, Regulators, algebraic cycles, and values of L-functions, in Algebraic K-theory and algebraic number theory, Contemporary Mathematics, vol. 83 (American Mathematical Society, Providence, RI, 1989), 183-310.

Sch93 A. J. Scholl, Extensions of motives, higher Chow groups and special values of L-functions, Progress in Mathematics, vol. 116 (Birkhäuser, Boston, MA, 1993), 279-292.

Tot92 B. Totaro, Milnor K-theory is the simplest part of algebraic K-theory, K-theory 6 (1992), 177-189.

Matt Kerr matkerr@math.uchicago.edu

Department of Mathematics, University of Chicago, Chicago, IL 60637, USA

James D. Lewis lewisjd@gpu.srv.ualberta.ca

Department of Mathematics, University of Alberta, Edmonton, Alberta, T6G 2G1, Canada

Stefan Müller-Stach mueller-stach@uni-mainz.de

Fachbereich 08, Institut für Mathematik, Johannes Gutenberg-Universität Mainz, 55099 Mainz,

Germany 